\title{
Universal Whitham hierarchy, dispersionless Hirota equations and multi-component KP hierarchy
}

\author{
Kanehisa Takasaki \\ Graduate School of Human and Environmental Studies, \\ Kyoto University \\ Yoshida, Sakyo, Kyoto, 606-8501, Japan \\ E-mail: takasaki@math.h.kyoto-u.ac.jp \\ Takashi Takebe \\ Department of Mathematics, Ochanomizu University \\ Otsuka 2-1-1, Bunkyo-ku, Tokyo, 112-8610, Japan \\ E-mail: takebe@math.ocha.ac.jp
}

\begin{abstract}
The goal of this paper is to identify the universal Whitham hierarchy of genus zero with a dispersionless limit of the multi-component $\mathrm{KP}$ hierarchy. To this end, the multi-component KP hierarchy is (re)formulated to depend on several discrete variables called "charges". These discrete variables play the role of lattice coordinates in underlying Toda field equations. A multi-component version of the so called differential Fay identity are derived from the Hirota equations of the $\tau$-function of this "charged" multi-component KP hierarchy. These multi-component differential Fay identities have a well-defined dispersionless limit (the dispersionless Hirota equations). The dispersionless Hirota equations turn out to be equivalent to the Hamilton-Jacobi equations for the $S$-functions of the universal Whitham hierarchy. The differential Fay identities themselves are shown to be a generating functional expression of auxiliary linear equations for scalar-valued wave functions of the multi-component KP hierarchy.
\end{abstract}


2000 Mathematics Subject Classification: 35Q58, 37K10, 58F07

Key words: universal Whitham hierarchy, dispersionless limit, Hirota equation, multi-component KP hierarchy

arXiv:nlin.SI/0608068 


\section{Introduction}

Nowadays most soliton equations are known to be (re)formulated in the form of Hirota equations for $\tau$-functions [1]. If such a soliton equation has a dispersionless limit (dispersionless integrable system), a natural question will be whether the Hirota equations have a reasonable dispersionless limit (dispersionless Hirota equations). This issue has been studied for the following relatively small number of cases (because the dispersionless integrable systems themselves are, at least currently, rare species).

1. The first example of dispersionless Hirota equations was discovered for the dispersionless KP hierarchy [2]. This dispersionless Hirota equation was obtained as a dispersionless limit of the so called differential Fay identity [3] (which actually turned out to be equivalent to the KP hierarchy itself). Another derivation using a Cauchy kernel was later developed [4].

2. Dispersionless Hirota equations are also known for the dispersionless Toda hierarchy, and applied to Laplacian growth [5, 6, 7, 8], WDVV associativity equations [9], string field theory [10, 11, 12, 13], etc. Moreover, a connection with some notions of complex analysis (the Grunsky coefficients and the Faber polynomials) was pointed out [14, 15]. Recently, a set of Fay-like identities for the Toda hierarchy were derived and shown to reduce to the dispersionless Hirota equations in the dispersionless limit [16].

3. A few other exotic soliton equation and some problems in complex analysis have been studied in the context of dispersionless Hirota equations [17, 18, 19, 20].

The goal of this paper is to enlarge this list to include a multi-component generalization of the KP hierarchy (multi-component KP hierarchy) [21, 22, 23, 24, 25].

Unlike the aforementioned cases, the multi-component KP hierarchy is usually formulated in a matrix form with matrix (pseudo)differential operators and vector (or matrix) valued wave functions. This yields technical difficulties when one naively attempts to give a prescription of dispersionless limit in the Lax formalism. As regards the multi-component KP hierarchy, thus, defining a reasonable dispersionless limit itself has been considered a tough problem.

These difficulties can be circumvented in two ways. One way is to resort to a scalar Lax formalism based on scalar operators and wave functions [26]. A 
prototype of such a scalar Lax formalism can be seen in the Toda hierarchy, which is equivalent to the two-component KP hierarchy that depends an extra discrete variable $s$ (i.e., the lattice coordinate) [27], and nevertheless has a scalar Lax formalism (in terms of difference operators in $s$ ). It is rather straightforward to generalize this point of view to the case with more than two components. The other way is to seek for a prescription of dispersionless limit for Hirota equations rather than the Lax formalism. Actually, these two approaches lead to the same result. In this paper, we primarily choose the route from Hirota equations, which eventually reaches the scalar Lax formalism as well.

In the case of the $N+1$-component KP hierarchy, we can introduce $N$ discrete variables $s_{1}, \ldots, s_{N}$. These variables stand for charges of a state in the Fock space of an $N+1$-component charged free fermion system in the field theoretical formalism [23, 25]. In analogy with the aforementioned interpretation of the Toda hierarchy, this charged multi-component KP hierarchy may be thought of as a generalization of the Toda hierarchy as well.

The dispersionless limit of this hierarchy turns out to be the universal Whitham hierarchy of genus zero. The universal Whitham hierarchy was introduced, at all genera, by Krichever [28] as a universal framework for both dispersionless integrable systems and Whitham modulation equations. The nonzero-genus cases were recently applied to Laplacian growth of multiplyconnected domains [29, 30]. The zero-genus case, too, is the target of recent studies [31, 32, 33, which have revealed interesting new aspects of this hierarchy. One can see from our results that many of them stem from the multi-component KP hierarchy.

This paper is organized as follows. Sections 2 is a review of the universal Whitham hierarchy of genus zero and some related equations. Of particular importance is the Hamilton-Jacobi equations satisfied by the $S$-functions. These equations play the role of auxiliary linear equations in usual soliton equations. In Section 3, we show that these equations can be converted to a generating functional form, which we shall later identify with dispersionless Hirota equations. Section 4 presents a brief account of the $\tau$-function of the "charged" multi-component KP hierarchy and the Hirota equations in a generating functional bilinear form. In Section 5 , we derive a set of differential Fay identities for this hierarchy, and show that their dispersionless limit (namely, the dispersionless Hirota equations in this case) coincide with the equations obtained in Section 3. In Section 6, we show that these differential Fay identities are in fact a generating functional expression of auxiliary linear equations in the aforementioned scalar Lax formalism. 


\section{Universal Whitham hierarchy of genus zero}

\subsection{Dynamical variables}

We consider the universal Whitham hierarchy of genus zero with $N+1$ marked points (or "punctures") on a Riemann sphere with coordinate $p$. One of these marked points is fixed at $p=\infty$, and the others are located at $p=q_{1}, \ldots, q_{N}$, which are part of dynamical variables. The other dynamical variables are the coefficients of Laurent series $z_{0}(p), z_{1}(p), \ldots, z_{N}(p)$ of the form

$$
\begin{aligned}
& z_{0}(p)=p+\sum_{j=2}^{\infty} u_{0 j} p^{-j+1}, \\
& z_{\alpha}(p)=\frac{r_{\alpha}}{p-q_{\alpha}}+\sum_{j=1}^{\infty} u_{\alpha j}\left(p-q_{\alpha}\right)^{j-1} \quad(\alpha=1, \ldots, N)
\end{aligned}
$$

defined at the marked points.

The hierarchy has $N+1$ sets of time variables $t_{0 n}(n=1,2, \ldots)$ and $t_{\alpha n}$ $(\alpha=1, \ldots, N, n=0,1, \ldots)$ attached to the marked points at $p=\infty$ and $p=q_{\alpha}(\alpha=1, \ldots, N)$, respectively. The lowest ones $t_{01}, t_{10}, \ldots, t_{N 0}$ play a special role. For convenience, we introduce the auxiliary variable

$$
t_{00}=-\sum_{\alpha=1}^{N} t_{\alpha 0}
$$

which is, of course, not an independent variable. Let $\partial_{\alpha n}$ denote the derivatives

$$
\partial_{\alpha n}=\partial / \partial t_{\alpha n}
$$

\subsection{Lax and Zakharov-Shabat equations}

Time evolutions of the hierarchy are generated by the dispersionless Lax equations

$$
\partial_{\alpha n} z_{\beta}(p)=\left\{\Omega_{\alpha n}(p), z_{\beta}(p)\right\}
$$

with respect to the Poisson bracket

$$
\{f, g\}=\frac{\partial f}{\partial p} \frac{\partial g}{\partial t_{01}}-\frac{\partial f}{\partial t_{01}} \frac{\partial g}{\partial p} .
$$


The "Hamiltonians" $\Omega_{\alpha n}(p)$ are defined as

$$
\begin{aligned}
& \Omega_{0 n}(p)=\left(z_{0}(p)^{n}\right)_{(0, \geq 0)}, \quad \Omega_{\alpha n}(p)=\left(z_{\alpha}(p)^{n}\right)_{(\alpha,>0)} \quad(n=1,2, \ldots), \\
& \Omega_{\alpha 0}(p)=-\log \left(p-q_{\alpha}\right),
\end{aligned}
$$

where ()$_{(0, \geq 0)}$ denotes the projection to nonnegative powers of $p$, and ()$_{(\alpha,>0)}$ the projection to positive powers of $\left(p-q_{\alpha}\right)^{-1}$. In other words,

$$
\begin{aligned}
& z_{0}(p)^{n}=\Omega_{0 n}(p)+O\left(p^{-1}\right) \quad(p \rightarrow \infty), \\
& z_{\alpha}(p)^{n}=\Omega_{\alpha n}(p)+O(1) \quad\left(p \rightarrow q_{\alpha}\right)
\end{aligned}
$$

for $n \geq 1$. In particular,

$$
\Omega_{01}(p)=p, \quad \Omega_{\alpha 1}(p)=\frac{r_{\alpha}}{p-q_{\alpha}} .
$$

The Hamiltonians $\Omega_{\alpha n}(p)$ satisfy the dispersionless Zakharov-Shabat equations

$$
\partial_{\beta n} \Omega_{\alpha m}(p)-\partial_{\alpha m} \Omega_{\beta n}(p)+\left\{\Omega_{\alpha m}(p), \Omega_{\beta n}(p)\right\}=0 .
$$

They can be converted to the equation

$$
\omega \wedge \omega=0
$$

of the closed 2-form

$$
\omega=\sum_{n=1}^{\infty} d \Omega_{0 n}(p) \wedge d t_{0 n}+\sum_{\alpha=1}^{N} \sum_{n=0}^{\infty} d \Omega_{\alpha n}(p) \wedge d t_{\alpha n} .
$$

By Darboux's theorem, $z_{\beta}(p)$ has a "conjugate" variable $\zeta_{\beta}(p)$ with which $\omega$ can be written in the "canonical form"

$$
\omega=d z_{\beta}(p) \wedge d \zeta_{\beta}(p)
$$

Consequently, $\zeta_{\beta}(p)$ satisfies the Lax equations

$$
\partial_{\alpha n} \zeta_{\beta}(p)=\left\{\Omega_{\alpha n}(p), \zeta_{\beta}(p)\right\}
$$

of the same form as $z_{\beta}(p)$, alongside the canonical Poisson commutation relation

$$
\left\{z_{\beta}(p), \zeta_{\beta}(p)\right\}=1 \text {. }
$$

Thus $\zeta_{\beta}(p)$ amounts to the Orlov-Schulman functions in the dispersionless KP hierarchy. 


\section{$2.3 \quad S$-functions}

We can rewrite (8) as

$$
d\left(\theta+\zeta_{\beta}(p) d z_{\beta}(p)\right)=0
$$

where

$$
\theta=\sum_{n=1}^{\infty} \Omega_{0 n}(p) d t_{0 n}+\sum_{\alpha=1}^{N} \sum_{n=0}^{\infty} \Omega_{\alpha n} d t_{\alpha n}
$$

This implies the existence of a function $\mathcal{S}_{\beta}(p)$ such that

$$
d \mathcal{S}_{\beta}(p)=\theta+\zeta_{\beta}(p) d z_{\beta}(p)
$$

or, more explicitly,

$$
d \mathcal{S}_{\beta}(p)=\zeta_{\beta}(p) d z_{\beta}(p)+\sum_{n=1}^{\infty} \Omega_{0 n}(p) d t_{0 n}+\sum_{\alpha=1}^{N} \sum_{n=0}^{\infty} \Omega_{\alpha n}(p) d t_{\alpha n} .
$$

$\mathcal{S}_{0}(p)$ and $\mathcal{S}_{\beta}(p)$ turn out to have Laurent expansion of the following form:

$$
\begin{aligned}
& \mathcal{S}_{0}(p)=\sum_{n=1}^{\infty} t_{0 n} z_{0}(p)^{n}+t_{00} \log z_{0}(p)-\sum_{n=1}^{\infty} \frac{z_{0}(p)^{-n}}{n} v_{0 n} \\
& \mathcal{S}_{\beta}(p)=\sum_{n=1}^{\infty} t_{\beta n} z_{\beta}(p)^{n}+t_{\beta 0} \log z_{\beta}(p)+\phi_{\beta}-\sum_{n=1}^{\infty} \frac{z_{\beta}(p)^{-n}}{n} v_{\beta n}
\end{aligned}
$$

where $v_{0 n}, v_{\alpha n}$ and $\phi_{\beta}$ are functions of the time variables.

These $S$-functions can also be written as

$$
\mathcal{S}_{0}(p)=S_{0}\left(z_{0}(p)\right), \quad \mathcal{S}_{\beta}(p)=S_{\beta}\left(z_{\beta}(p)\right)
$$

where $S_{0}(z)$ and $S_{\beta}(z)$ are defined as

$$
\begin{aligned}
& S_{0}(z)=\sum_{n=1}^{\infty} t_{0 n} z^{n}+t_{00} \log z-\sum_{n=1}^{\infty} \frac{z^{-n}}{n} v_{0 n}, \\
& S_{\beta}(z)=\sum_{n=1}^{\infty} t_{\beta n} z^{n}+t_{\beta 0} \log z+\phi_{\beta}-\sum_{n=1}^{\infty} \frac{z^{-n}}{n} v_{\beta n} .
\end{aligned}
$$

The latter functions, too, are called $S$-functions, which play a more fundamental role in the subsequent consideration. 
As (11) implies, differentiating (12) by $z_{\beta}(p)$ while leaving the time variables constant yields $\zeta_{0}(p)$ and $\zeta_{\beta}(p)$, which thus turns out to be Laurent series of the form

$$
\begin{aligned}
& \zeta_{0}(p)=\sum_{n=1}^{\infty} n t_{0 n} z_{0}(p)^{n-1}+\frac{t_{00}}{z_{0}(p)}+\sum_{n=1}^{\infty} z_{0}(p)^{-n-1} v_{0 n}, \\
& \zeta_{\beta}(p)=\sum_{n=1}^{\infty} n t_{\alpha n} z_{\beta}(p)^{n-1}+\frac{t_{\beta 0}}{z_{\beta}(p)}+\sum_{n=1}^{\infty} z_{\beta}(p)^{-n} v_{\beta n} .
\end{aligned}
$$

Similarly, differentiating (12) by the time variables gives rise to the following expressions of $\Omega_{\alpha n}(p)$ 's:

$$
\begin{aligned}
& \Omega_{0 n}(p)=\left\{\begin{array}{l}
z_{0}(p)^{n}-\sum_{m=1}^{\infty} \frac{z_{0}(p)^{-m}}{m} \partial_{0 n} v_{0 m}, \\
\partial_{0 n} \phi_{\beta}-\sum_{m=1}^{\infty} \frac{z_{\beta}(p)^{-m}}{m} \partial_{0 n} v_{\beta m},
\end{array}\right. \\
& \Omega_{\alpha n}(p)=\left\{\begin{array}{l}
-\delta_{n 0} \log z_{0}(p)-\sum_{m=1}^{\infty} \frac{z_{0}(p)^{-m}}{m} \partial_{\alpha 0} v_{0 m}, \\
\delta_{\alpha \beta} \log z_{\beta}(p)+\partial_{\alpha 0} \phi_{\beta}-\sum_{m=1}^{\infty} \frac{z_{\beta}(p)^{-m}}{m} \partial_{\alpha 0} v_{\beta m} .
\end{array}\right.
\end{aligned}
$$

\subsection{Hamilton-Jacobi equations}

Since $\Omega_{01}(p)=p$, the foregoing expression of $\Omega_{01}(p)$ implies that $z_{0}(p)$ and $z_{\beta}(p)$ satisfy the equations

$$
\begin{aligned}
& p=z_{0}(p)-\sum_{m=1}^{\infty} \frac{z_{0}(p)^{-m}}{m} \partial_{01} v_{0 m}, \\
& p=\partial_{01} \phi_{\beta}-\sum_{m=1}^{\infty} \frac{z_{\beta}(p)^{-m}}{m} \partial_{01} v_{\beta m} .
\end{aligned}
$$

In other words, the inverse functions $p=p_{0}(z)$ and $p=p_{\beta}(z)$ of $z=z_{0}(p)$ and $z=z_{\beta}(p)$ are given explicitly by

$$
\begin{array}{r}
p_{0}(z)=z-\sum_{m=1}^{\infty} \frac{z^{-m}}{m} \partial_{01} v_{0 m}=\partial_{01} S_{0}(z), \\
p_{\beta}(z)=\partial_{01} \phi_{\beta}-\sum_{m=1}^{\infty} \frac{z^{-m}}{m} \partial_{01} v_{\beta m}=\partial_{01} S_{\beta}(z) .
\end{array}
$$


As a byproduct of these relations, we find an expression of $p_{\beta}$ and $r_{\beta}$ in terms of the coefficients of $S_{\beta}(z)$ : Since $z_{\beta}(p)$ is assumed to have Laurent expansion of the form

$$
z_{\beta}(p)=\frac{r_{\beta}}{p-q_{\beta}}+O(1)
$$

at $p=q_{\beta}$, the inverse function should have Laurent expansion of the form

$$
p_{\beta}(z)=q_{\beta}+r_{\beta} z^{-1}+O\left(z^{-2}\right) .
$$

Comparing this with (1), we readily find that

$$
q_{\beta}=\partial_{01} \phi_{\beta}, \quad r_{\beta}=-\partial_{01} v_{\beta 1} .
$$

We can now derive a system of Hamilton-Jacobi type for the $S$-functions. This is achieved by substituting $p=p_{\beta}(z)(\beta=0,1, \ldots, N)$ in (11). The outcome of substitution is the equation

$$
d S_{\beta}(z)=S_{\beta}^{\prime}(z) d z+\sum_{n=1}^{\infty} \Omega_{0 n}\left(p_{\beta}(z)\right) d t_{0 n}+\sum_{\alpha=1}^{N} \sum_{n=0}^{\infty} \Omega_{\alpha n}\left(p_{\beta}(z)\right) d t_{\alpha n},
$$

which implies that

$$
\partial_{\alpha n} S_{\beta}(z)=\Omega_{\alpha n}\left(p_{\beta}(z)\right) .
$$

Since $p_{\beta}(z)$ is related to the $S$-functions as (18) shows, we eventually obtain the Hamilton-Jacobi equations

$$
\partial_{\alpha n} S_{\beta}(z)=\Omega_{\alpha n}\left(\partial_{01} S_{\beta}(z)\right) .
$$

The lowest Hamilton-Jacobi equations have a special meaning. Whereas the equation for $t_{01}$ is trivial, those for $t_{\alpha 0}(\alpha=1, \ldots, N)$ read

$$
\partial_{\alpha 0} S_{\beta}(z)=-\log \left(\partial_{01} S_{\beta}(z)-q_{\alpha}\right),
$$

which can be solved for $p_{\beta}(z)=\partial_{01} S_{\beta}(z)$ as

$$
p_{\beta}(z)=e^{-\partial_{\alpha 0} S_{\beta}(z)}+q_{\alpha} .
$$

In particular, choosing $\beta=\alpha$ and extracting the coefficient of $z^{-1}$, we find another expression of $r_{\alpha}$ :

$$
r_{\alpha}=e^{-\partial_{\alpha 0} \phi_{\alpha}} .
$$

These Hamilton-Jacobi equations characterize the $S$-functions in their own terms. One can recover the previous setting of the universal Whitham hierarchy by just changing variables from $z$ to $p$. In this sense, the two systems are two different pictures of the same system, and one can move from one picture to the other by (11) and (18). 


\section{$2.5 \quad F$-function}

Following Martínez Alonso, Mañas and Medina [33], we define the $F$-function (the logarithm of the dispersionless $\tau$-function [28]) to be a solution of the following equations:

$$
\begin{aligned}
& \partial_{0 n} F=v_{0 n}, \quad \partial_{\alpha n} F=v_{\alpha n} \quad(n=1,2, \ldots), \\
& \partial_{\alpha 0} F=-\phi_{\alpha}+\sum_{\beta=1}^{\alpha} t_{\beta 0} \log (-1) \quad(\alpha=1, \ldots, N),
\end{aligned}
$$

where $\log (-1)$ is understood to be equal to, say, $\pi i$, though the choice of the branch is irrelevant in the final result. Now we can rewrite (13) as

$$
\begin{aligned}
& S_{0}(z)=\sum_{n=1}^{\infty} t_{0 n} z^{n}+t_{00} \log z-D_{0}(z) F \\
& S_{\alpha}(z)=\sum_{n=1}^{\infty} t_{\alpha n} z^{n}+t_{\alpha 0} \log z+\phi_{\alpha}-D_{\alpha}(z) F,
\end{aligned}
$$

where $D_{0}(z)$ and $D_{\alpha}(z)$ denotes the following differential operators:

$$
D_{0}(z)=\sum_{n=1}^{\infty} \frac{z^{-n}}{n} \partial_{0 n}, \quad D_{\alpha}(z)=\sum_{n=1}^{\infty} \frac{z^{-n}}{n} \partial_{\alpha n} .
$$

It should be mentioned that the last part of (25) is slightly different from that of Mañas et al. [33. Their proof of consistency of the defining equations, however, persists to be valid in this form as well. We have modified their definition so as to match the fermionic formula of the tau functions by Date et al. [23].

\section{Dispersionless Hirota equations as gener- ating functional form of Hamilton-Jacobi equations}

\subsection{Faber polynomials}

Following Teo's idea [15] developed for the case of the dispersionless KP and Toda hierarchies, we now consider the notion of Faber polynomials of $p_{0}(z)$ and $p_{\alpha}(z)$, and show that they actually coincide with $\Omega_{0 n}(p)$ and $\Omega_{\alpha n}(p)$. 
The Faber polynomials $\Phi_{0 n}(p)$ and $\Phi_{\alpha n}(p)$ of $p_{0}(z)$ and $p_{\alpha}(z)$ are defined by the following generating functions:

$$
\begin{aligned}
& \log \frac{p_{0}(z)-q}{z}=-\sum_{n=1}^{\infty} \frac{z^{-n}}{n} \Phi_{0 n}(q), \\
& \log \frac{q-p_{\alpha}(z)}{q-q_{\alpha}}=-\sum_{n=1}^{\infty} \frac{z^{-n}}{n} \Phi_{\alpha n}(q) .
\end{aligned}
$$

Rewriting the left hand side as

$$
\log \frac{p_{0}(z)-q}{z}=\log \frac{p_{0}(z)}{z}+\log \left(1-\frac{q}{p_{0}(z)}\right)
$$

and

$$
\log \frac{q-p_{\alpha}(z)}{q-q_{\alpha}}=\log \left(1-\frac{p_{\alpha}(z)-q_{\alpha}}{q-q_{\alpha}}\right)
$$

and recalling that

$$
p_{0}(z)=z+O\left(z^{-1}\right), \quad p_{\alpha}(z)=q_{\alpha}+O\left(z^{-1}\right),
$$

we readily see that

1) $\Phi_{0 n}(q)$ is a monic polynomial of $q$, and

2) $\Phi_{\alpha n}(q)$ is a polynomial of $\left(q-q_{\alpha}\right)^{-1}$ with no constant term.

Lemma 1. The Faber polynomials coincide with $\Omega_{0 n}(p)$ and $\Omega_{\alpha n}(p)$ :

$$
\Phi_{0 n}(q)=\Omega_{0 n}(q), \quad \Phi_{\alpha n}(q)=\Omega_{\alpha n}(q) .
$$

Proof. A more explicit expression of these polynomials can be obtained by differentiating the generating functions by $z$ as

$$
\begin{aligned}
\frac{p_{0}^{\prime}(z)}{p_{0}(z)-q}-\frac{1}{z} & =\sum_{n=1}^{\infty} z^{-n-1} \Phi_{0 n}(q), \\
\frac{p_{\alpha}^{\prime}(z)}{p_{\alpha}(z)-q} & =\sum_{n=1}^{\infty} z^{-n-1} \Phi_{\alpha n}(q)
\end{aligned}
$$

and extracting the Laurent coefficients by contour integrals. As regards $\Phi_{0 n}(z)$, this yields the contour integral formula

$$
\Phi_{0 n}(q)=\frac{1}{2 \pi i} \oint \frac{z^{n} p_{0}^{\prime}(z) d z}{p_{0}(z)-q}=\frac{1}{2 \pi i} \oint_{|p|=R} \frac{z_{0}(p)^{n} d p}{p-q},
$$


where the contour $|p|=R$ in the second integral is understood to be sufficiently large and to encircle $q$ anti-clockwise; the contour in the first integral is its image under the mapping $p \mapsto z_{0}(p)$. The last contour integral is nothing but the polynomial part of $z_{0}(p)^{n}$ evaluated at $q$, i.e., $\Omega_{0 n}(q)$. In much the same way, we have the contour integral formula

$$
\Phi_{\alpha n}(q)=\frac{1}{2 \pi i} \oint \frac{z^{n} p_{\alpha}^{\prime}(z) d z}{p_{\alpha}(z)-q}=\frac{1}{2 \pi i} \oint_{\left|p-q_{\alpha}\right|=r} \frac{z_{\alpha}(p)^{n} d p}{p-q},
$$

where the contour $\left|p-q_{\alpha}\right|=r$ in the second integral is chosen to be sufficiently small and to encircle $q_{\alpha}$ clockwise, leaving $q$ outside. This contour integral leaves negative powers of the Laurent expansion of $z_{\alpha}(p)^{n}$ at $p=q_{\alpha}$, hence coincides with $\Omega_{\alpha n}(q)$.

Bearing this interpretation of $\Omega_{\alpha n}(p)$ 's in mind, we turn to the HamiltonJacobi equations (21). The goal is to transform these equations, more precisely, those for $n \geq 1$, into a generating functional form.

\subsection{First subset of Hamilton-Jacobi equations}

Let us first consider the equations for $\alpha=0, \beta=0$ and $n \geq 1$ :

$$
\partial_{0 n} S_{0}(z)=\Omega_{0 n}\left(\partial_{01} S_{0}(z)\right)=\Omega_{0 n}\left(p_{0}(z)\right) .
$$

Lemma 2. (29) is equivalent to

$$
\log \frac{p_{0}(z)-p_{0}(w)}{z-w}=D_{0}(z) D_{0}(w) F
$$

Proof. Substituting $q=p_{0}(w)$ in the generating function of the Faber polynomials $\Phi_{0 n}(q)=\Omega_{0 n}(q)$ yields the identity

$$
\log \frac{p_{0}(z)-p_{0}(w)}{z}=-\sum_{n=1}^{\infty} \frac{z^{-n}}{n} \Omega_{0 n}\left(p_{0}(w)\right) .
$$

Therefore (29) can be cast into the generating functional form

$$
\log \frac{p_{0}(z)-p_{0}(w)}{z}=-\sum_{n=1}^{\infty} \frac{z^{-m}}{m} \partial_{0 n} S_{0}(w) .
$$

(26) implies that $\partial_{0 n} S_{0}(w)$ on the right hand side can be expressed as

$$
\partial_{0 n} S_{0}(w)=w^{n}-\sum_{m=1}^{\infty} \frac{w^{-m}}{m} \partial_{0 n} \partial_{0 m} F .
$$


Thus the generating functional equation can be rewritten as

$$
\begin{aligned}
\log \frac{p_{0}(z)-p_{0}(w)}{z} & =-\sum_{n=1}^{\infty} \frac{z^{-n}}{n}\left(w^{n}-\sum_{m=1}^{\infty} \frac{w^{-m}}{m} \partial_{0 n} \partial_{0 m} F\right) \\
& =\log \left(1-\frac{w}{z}\right)+D_{0}(z) D_{0}(w) F
\end{aligned}
$$

Moving the first term on the right hand side to the left hand side, we obtain (30) as a generating functional form of (29).

\subsection{Second subset of Hamilton-Jacobi equations}

The second subset of equations are those for $\alpha=1, \ldots, N, \beta=0$, and $n \geq 1$ :

$$
\partial_{\alpha n} S_{0}(z)=\Omega_{\alpha n}\left(\partial_{01} S_{0}(z)\right)=\Omega_{\alpha n}\left(p_{0}(z)\right) .
$$

Lemma 3. (31) is equivalent to

$$
\log \frac{p_{0}(z)-p_{\beta}(w)}{p_{0}(z)-q_{\beta}}=D_{0}(z) D_{\beta}(w) F
$$

Proof. Substituting $q=p_{0}(w)$ in the generating function of the Faber polynomials $\Phi_{\alpha n}(q)=\Omega_{\alpha n}(q)$ leads to the identity

$$
\log \frac{p_{0}(w)-p_{\alpha}(z)}{p_{0}(w)-q_{\alpha}}=-\sum_{n=1}^{\infty} \frac{z^{-n}}{n} \Omega_{\alpha n}\left(p_{0}(w)\right) .
$$

Therefore (31) can be converted to the generating functional form

$$
\log \frac{p_{0}(w)-p_{\alpha}(z)}{p_{0}(w)-q_{\alpha}}=-\sum_{n=1}^{\infty} \partial_{\alpha n} S_{0}(w) .
$$

(26) implies that $\partial_{\alpha n} S_{0}(w)$ on the right hand side can be rewritten as

$$
\partial_{\alpha n} S_{0}(w)=-\sum_{m=1}^{\infty} \frac{w^{-m}}{m} \partial_{\alpha n} \partial_{0 m} F
$$

Thus the generating functional equation turns into (31). 


\subsection{Third subset of Hamilton-Jacobi equations}

Let us now consider the equations for $\alpha=0, \beta=1, \ldots, N$ and $n \geq 1$ :

$$
\partial_{0 n} S_{\beta}(z)=\Omega_{0 n}\left(\partial_{01} S_{\beta}(z)\right)=\Omega_{0 n}\left(p_{\beta}(z)\right) .
$$

Unlike the previous two cases, we now have to use (22) to rewrite these Hamilton-Jacobi equations.

Lemma 4. If (22) is satisfied, (33) is equivalent to (32).

Proof. As in the case of (29), we can convert (33) to the generating functional form

$$
\log \frac{p_{0}(z)-p_{\beta}(w)}{z}=-\sum_{n=1}^{\infty} \frac{z^{-n}}{n} \partial_{0 n} S_{\beta}(w)
$$

By (26), we can rewrite $\partial_{0 n} S_{\beta}(w)$ on the right hand side as

$$
\partial_{0 n} S_{\beta}(w)=-\partial_{0 n} \partial_{\beta 0} F+\sum_{m=1}^{\infty} \frac{w^{-m}}{m} \partial_{0 n} \partial_{\beta m} F
$$

This leads to an equation of the form

$$
\log \frac{p_{0}(z)-p_{\beta}(w)}{z}=\sum_{n=1}^{\infty} \frac{z^{-n}}{n} \partial_{0 n} \partial_{\beta 0} F+\sum_{n, m=1}^{\infty} \frac{z^{-n} w^{-m}}{n m} \partial_{0 n} \partial_{\beta m} F .
$$

The first sum on the right hand side can be evaluated as

$$
\begin{aligned}
\sum_{n=1}^{\infty} \frac{z^{-n}}{n} \partial_{0 n} \partial_{\beta 0} F & =\partial_{\beta 0} \sum_{n=1}^{\infty} \frac{z^{-n}}{n} \partial_{0 n} F \\
& =\partial_{\beta 0}\left(-S_{0}(z)+t_{00} \log z\right) \\
& =-\partial_{\beta 0} S_{0}(z)-\log z
\end{aligned}
$$

If (22) is satisfied, we can rewrite $\partial_{\beta_{0}} S_{0}(z)$ as

$$
\partial_{\beta 0} S_{0}(z)=-\log \left(p_{\beta}(z)-q_{\beta}\right)
$$

and obtain (32). 


\subsection{Fourth subset of Hamilton-Jacobi equations}

The fourth subset consists of the Hamilton-Jacobi equations for $\alpha=\beta=$ $1, \ldots, N$ and $n \geq 1$ :

$$
\partial_{\alpha n} S_{\alpha}(z)=\Omega_{\alpha n}\left(\partial_{01} S_{\alpha}(z)\right)=\Omega_{\alpha n}\left(p_{\alpha}(z)\right) .
$$

Lemma 5. If (22) are satisfied, (34) is equivalent to

$$
\log \frac{p_{\alpha}(w)-p_{\alpha}(z)}{(z-w)\left(p_{\alpha}(z)-q_{\alpha}\right)\left(p_{\alpha}(w)-q_{\alpha}\right)}=\partial_{\alpha 0} \phi_{\alpha}+D_{\alpha}(z) D_{\alpha}(w) F
$$

Proof. As in the case of (31), we convert (34) to the generating functional form

$$
\log \frac{p_{\alpha}(w)-p_{\alpha}(z)}{p_{\alpha}(w)-q_{\alpha}}=-\sum_{n=1}^{\infty} \frac{z^{-n}}{n} \partial_{\alpha n} S_{\alpha}(w)
$$

and use (26) to rewrite $\partial_{\alpha n} S_{\alpha}(w)$ on the right hand side as

$$
\partial_{\alpha n} S_{\alpha}(w)=w^{n}-\partial_{\alpha n} \partial_{\alpha 0} F-\sum_{m=1}^{\infty} \frac{w^{-m}}{m} \partial_{\alpha n} \partial_{\alpha m} F .
$$

This leads us to an equation of the form

$$
\begin{aligned}
& \log \frac{p_{\alpha}(w)-p_{\alpha}(z)}{p_{\alpha}(w)-q_{\alpha}} \\
& =\log \left(1-\frac{w}{z}\right)+\sum_{n=1}^{\infty} \frac{z^{-n}}{n} \partial_{\alpha n} \partial_{\alpha 0} F+\sum_{n, m=1}^{\infty} \frac{z^{-n} w^{-m}}{n m} \partial_{\alpha n} \partial_{\alpha 0} F .
\end{aligned}
$$

The first sum on the right hand side can be evaluated as

$$
\begin{aligned}
\sum_{n=1}^{\infty} \frac{z^{-n}}{n} \partial_{\alpha n} \partial_{\alpha 0} F & =\partial_{\alpha 0}\left(\sum_{n=1}^{\infty} \frac{z^{-n}}{n} \partial_{\alpha n} F\right) \\
& =\partial_{\alpha 0}\left(-S_{\alpha}(z)+\sum_{n=1}^{\infty} t_{\alpha n} z^{n}+t_{\alpha 0} \log z+\partial_{\alpha 0} \phi_{\alpha}\right) \\
& =-\partial_{\alpha 0} S_{\alpha}(z)+\log z+\partial_{\alpha 0} \phi_{\alpha}
\end{aligned}
$$

As regards $\partial_{\alpha 0} S_{\alpha}(z)$, (22) implies that

$$
\partial_{\alpha 0} S_{\alpha}(z)=-\log \left(p_{\alpha}(z)-q_{\alpha}\right) .
$$

We now move all logarithmic terms to the left hand side, and obtain (35) as a generating functional form of (34). 


\subsection{Fifth subset of Hamilton-Jacobi equations}

The fifth subset of equations are those for $\alpha, \beta=1, \ldots, N, \alpha \neq \beta$, and $n \geq 1$ :

$$
\partial_{\alpha n} S_{\beta}(z)=\Omega_{\alpha n}\left(\partial_{01} S_{\beta}(z)\right)=\Omega_{\alpha n}\left(p_{\beta}(z)\right) \quad(\alpha \neq \beta) .
$$

Lemma 6. If (22) is satisfied, (36) is equivalent to

$$
\log \frac{p_{\beta}(w)-p_{\alpha}(z)}{\left(p_{\beta}(w)-q_{\alpha}\right)\left(p_{\alpha}(z)-q_{\beta}\right)}=\partial_{\beta 0} \phi_{\alpha}+D_{\alpha}(z) D_{\beta}(w) F
$$

We first convert these equations to the generating functional form

$$
\log \frac{p_{\beta}(w)-p_{\alpha}(z)}{p_{\beta}(w)-q_{\alpha}}=-\sum_{n=1}^{\infty} \frac{z^{-n}}{n} \partial_{\alpha n} S_{\beta}(w)
$$

and use (26) to rewrite the derivatives on the right hand side as

$$
\partial_{\alpha n} S_{\beta}(w)=-\partial_{\alpha n} \partial_{\beta 0} F-\sum_{m=1}^{\infty} \frac{w^{-m}}{m} \partial_{\alpha n} \partial_{\beta m} F .
$$

The outcome is an equation of the form

$$
\log \frac{p_{\beta}(w)-p_{\alpha}(z)}{p_{\beta}(w)-q_{\alpha}}=\sum_{n=1}^{\infty} \frac{z^{-n}}{n} \partial_{\alpha n} \partial_{\beta 0} F+\sum_{n, m=1}^{\infty} \frac{z^{-n} w^{-m}}{n m} \partial_{\alpha n} \partial_{\beta m} F .
$$

The first sum on the right hand side can be evaluated in the same way as the previous case, namely,

$$
\begin{aligned}
\sum_{n=1}^{\infty} \frac{z^{-n}}{n} \partial_{\alpha n} \partial_{\beta 0} F & =\partial_{\beta 0}\left(\sum_{n=1}^{\infty} \frac{z^{-n}}{n} \partial_{\alpha n} F\right) \\
& =-\partial_{\beta 0} S_{\alpha}(z)+\partial_{\beta 0} \phi_{0} \\
& =\log \left(p_{\alpha}(z)-q_{\beta}\right)+\partial_{\beta 0} \phi_{\alpha}
\end{aligned}
$$

where (22) has been used in such a form as

$$
\partial_{\beta 0} S_{\alpha}(z)=-\log \left(p_{\alpha}(z)-q_{\beta}\right) .
$$

Consequently, we have (37) as a generating functional form of (36). 


\subsection{Dispersionless Hirota equations}

We have thus converted the $n \neq 0$ sector of the full Hamilton-Jacobi equations (21) to the four generating functional equations (30), (32), (35) and (37). Since the $n=0$ sector (22) of the Hamilton-Jacobi equations is not included therein, these generating functional equations are still incomplete.

As the following theorem shows, we can modify these generating functional equations to include (22) as well. The complete generating functional equations, (38)-(41), resemble the dispersionless Hirota equations for the $\mathrm{KP}$ and Toda hierarchies. As we shall show later, these equations can be derived from bilinear equations of the $\tau$-function of the multi-component KP hierarchy. For these reasons, we call these equations "dispersionless Hitota equations."

Theorem 1. The full Hamilton-Jacobi equations (21) are equivalent to the following dispersionless Hirota equations:

$$
\begin{aligned}
e^{D_{0}(z) D_{0}(w) F} & =1-\frac{\partial_{01}\left(D_{0}(z)-D_{0}(w)\right) F}{z-w} \\
z e^{D_{0}(z)\left(\partial_{\alpha 0}+D_{\alpha}(w)\right) F} & =z-\partial_{01}\left(D_{0}(z)-\partial_{\alpha 0}-D_{\alpha}(w)\right) F \\
e^{\left(\partial_{\alpha 0}+D_{\alpha}(z)\right)\left(\partial_{\alpha 0}+D_{\alpha}(w)\right) F} & =-\frac{z w \partial_{01}\left(D_{\alpha}(z)-D_{\alpha}(w)\right) F}{z-w} \\
\epsilon_{\alpha \beta} e^{\left(\partial_{\alpha 0}+D_{\alpha}(z)\right)\left(\partial_{\beta 0}+D_{\beta}(w)\right) F} & =-\partial_{01}\left(\partial_{\alpha 0}+D_{\alpha}(z)-\partial_{\beta 0}-D_{\beta}(w)\right) F
\end{aligned}
$$

where $\epsilon_{\alpha \beta}$ is a sign factor defined as

$$
\epsilon_{\alpha \beta}=\epsilon_{\alpha \beta}(\mathbf{0})= \begin{cases}+1 & (\alpha \leq \beta), \\ -1 & (\alpha>\beta) .\end{cases}
$$

Proof. Because of the previous lemmas, we have only to show that the generating functional form of the Hamilton-Jacobi equations, upon supplemented by the $n=0$ sector (22), is equivalent to the dispersionless Hirota equations. As we show below, the four generating functional equations (30), (32), (35) and (37) respectively correspond to the four parts (38), (39), (40) and (41) of the dispersionless Hirota equations.

$(\underline{30}) \Leftrightarrow(\underline{38}) \quad$ To derive (38) from (30), we exponentiate the latter as

$$
p_{0}(z)-p_{0}(w)=1-\frac{e^{D_{0}(z) D_{0}(w) F}}{z-w}
$$

and substitute

$$
p_{0}(z)=z-\partial_{01} D_{0}(z) F, \quad p_{0}(w)=z-\partial_{01} D_{0}(w) F
$$

(see (18) and (26) ). This procedure is obviously reversible. 
$(32) \Rightarrow(39) \quad$ We exponentiate (32) as

$$
p_{0}(z)-p_{\beta}(w)=\left(p_{0}(z)-q_{\beta}\right) e^{D_{0}(z) D_{\beta}(w) F}
$$

and examine the both hand sides. As regards the left hand side, we substitute

$$
p_{0}(z)=z-\partial_{01} D_{0}(z) F, \quad p_{\beta}(w)=-\partial_{01} \partial_{\beta 0} F-\partial_{01} D_{\beta}(w) F
$$

and find that

$$
\text { LHS }=z-\partial_{01}\left(D_{0}(z)-\partial_{\beta 0}-D_{\beta}(w)\right) F .
$$

As regards the right hand side, we use the relation

$$
p_{0}(z)-q_{\beta}=e^{-\partial_{\beta 0} S_{0}(z)}=z e^{\partial_{\beta 0} D_{0}(z) F}
$$

implied by (23) and (26). Thus we obtain (39).

$(\mathbf{3 9}) \Rightarrow(\mathbf{3 2}) \quad$ Taking the limit as $w \rightarrow \infty$ in (39), we obtain the relation

$$
z-\partial_{01}\left(D_{0}(z)-\partial_{\beta 0}\right) F=z e^{\partial_{\beta 0} D_{0}(z) F}=e^{-\partial_{\beta 0} S_{0}(z)} .
$$

Since (19) and (25) imply that

$$
\partial_{01} \partial_{\beta 0} F=-q_{\beta}, \quad p_{0}(z)=z-\partial_{01} D_{0}(z) F,
$$

we can rewrite this relation as

$$
p_{0}(z)-q_{\beta}=e^{-\partial_{\beta 0} S_{0}(z)},
$$

which is equivalent to one of the equations of (22). Having this equation, we can recover (32) from (39).

$(\underline{35}) \Rightarrow(\underline{40}) \quad$ We rewrite $(\underline{35})$ as

$$
\frac{p_{\alpha}(w)-p_{\alpha}(z)}{z-w}=\left(p_{\alpha}(z)-q_{\alpha}\right)\left(p_{\alpha}(w)-q_{\alpha}\right) \exp \left(\partial_{\alpha 0} \phi_{\alpha}+D_{\alpha}(z) D_{\alpha}(w) F\right)
$$

and examine the both hand sides. The left hand side can be processed in the same way as in the preceding cases:

$$
\operatorname{LHS}=\frac{\partial_{01}\left(D_{\alpha}(z)-D_{\alpha}(w)\right) F}{z-w} .
$$

As regards the right hand side, we use the relation

$$
p_{\alpha}(z)-q_{\alpha}=e^{-\partial_{\alpha 0} S_{\alpha}(z)}=z^{-1} e^{-\partial_{\alpha 0} \phi_{\alpha}+\partial_{\alpha 0} D_{\alpha}(z) F}
$$


and its counterpart for $p_{\alpha}(w)-q_{\alpha}$ to rewrite it as

$\mathrm{RHS}=z^{-1} w^{-1} \exp \left(-\partial_{\alpha 0} \phi+\partial_{\alpha 0} D_{\alpha}(z) F+\partial_{\alpha 0} D_{\alpha}(w) F+D_{\alpha}(z) D_{\alpha}(w) F\right)$.

Since (25) implies that

$$
\partial_{\alpha 0} \phi_{\alpha}=-\partial_{\alpha 0}^{2} F+\log (-1),
$$

we can further rewrite the foregoing expression as

$$
\begin{aligned}
\mathrm{RHS} & =-z^{-1} w^{-1} \exp \left(\partial_{\alpha 0}^{2} F+\partial_{\alpha 0} D_{\alpha}(z) F+\partial_{\alpha 0} D_{\alpha}(w) F+D_{\alpha}(z) D_{\alpha}(w) F\right) \\
& =-z^{-1} w^{-1} e^{\left(\partial_{\alpha 0}+D_{\alpha}(z)\right)\left(\partial_{\alpha 0}+D_{\alpha}(w)\right) F} .
\end{aligned}
$$

This completes the derivation of (40).

(40) $\Rightarrow$ (35) Taking the limit as $w \rightarrow \infty$ in (40) leads to the equation

$$
\partial_{01} D_{\alpha}(z) F=e^{\left(\partial_{\alpha 0}+D_{\alpha}(z)\right) \partial_{\alpha 0} F} .
$$

Since

$$
\partial_{01} \phi_{\alpha}=q_{\alpha}, \quad \partial_{\alpha 0}^{2} F=-\partial_{\alpha 0} \phi+\log (-1),
$$

we can rewrite the last equation as

$$
p_{\alpha}(z)-q_{\alpha}=e^{-\partial_{\alpha 0} \phi_{\alpha}-\partial_{\alpha 0} D_{\alpha}(z) F}=e^{-\partial_{\alpha 0} S_{\alpha}(z)} .
$$

This is equivalent to one of the equations of (22). We can thereby recover (35).

$(\underline{37}) \Rightarrow$ (41) We first exponentiate (37) as

$$
p_{\beta}(w)-p_{\alpha}(z)=\left(p_{\beta}(w)-q_{\alpha}\right)\left(p_{\alpha}(z)-q_{\beta}\right) e^{\partial_{\beta 0} \phi_{\alpha}+D_{\alpha}(z) D_{\beta}(w) F} .
$$

Repeating almost the same calculations as the previous cases, we can rewrite the left hand side as

$$
\text { LHS }=\partial_{01}\left(\partial_{\alpha 0}+D_{\alpha}(z)-\partial_{\beta 0}-D_{\beta}(w)\right) F .
$$

As regards the right hand side, we use the relation

$$
p_{\alpha}(z)-q_{\beta}=e^{-\partial_{\beta 0} S_{\alpha}(z)}=e^{-\partial_{\beta 0} \phi_{\alpha}+\partial_{\beta 0} D_{\alpha}(z) F}
$$

and its counterpart for $p_{\beta}(w)-q_{\alpha}$ implied by (23) and (26) to find that

$$
\mathrm{RHS}=\exp \left(-\partial_{\alpha 0} \phi_{\beta}-\partial_{\alpha 0} \partial_{\beta 0} F+\left(\partial_{\alpha 0}+D_{\alpha}(z)\right)\left(\partial_{\beta 0}+D_{\beta}(w)\right) F\right) .
$$


On the other hand, (25) implies that

$$
\partial_{\alpha 0} \phi_{\beta}+\partial_{\alpha 0} \partial_{\beta 0} F= \begin{cases}\log (-1) & (\alpha \leq \beta), \\ 0 & (\alpha>\beta),\end{cases}
$$

so that

$$
\exp \left(-\partial_{\beta 0} \phi_{\alpha}-\partial_{\alpha 0} \partial_{\beta 0} F\right)=-\epsilon_{\alpha \beta} .
$$

We can thus derive (41) from (37).

(41) $\Rightarrow$ (37) This is mostly parallel to the previous cases. We take the limit as $w \rightarrow \infty$ in (41). The outcome is the equation

$$
-\partial_{01}\left(\partial_{\alpha 0}+D_{\alpha}(z)-\partial_{\beta 0}\right) F=\epsilon_{\alpha \beta} e^{\left(\partial_{\alpha 0}+D_{\alpha}(z)\right) \partial_{\beta 0} F} .
$$

It is now easy to see that the left hand side is equal to $p_{\alpha}(z)-q_{\beta}$. As regards the right hand side, we can use the aforementioned relation

$$
\exp \left(-\partial_{\beta 0} \phi_{\alpha}-\partial_{\alpha 0} \partial_{\beta 0} F\right)=-\epsilon_{\alpha \beta}
$$

once again to rewrite it as

$$
\epsilon_{\alpha \beta} e^{\partial_{\beta 0} \partial_{\alpha 0} F+\partial_{\beta 0} \phi_{\alpha}} e^{-\partial_{\beta 0} S_{\alpha}(z)}=-e^{-\partial_{\beta 0} S_{\alpha}(z)} .
$$

Thus the foregoing equation boils down to

$$
p_{\alpha}(z)-q_{\beta}=e^{-\partial_{\beta 0} S_{\alpha}(z)} .
$$

We can thereby recover (37).

\section{Tau function of mutli-component KP hier- archy}

\subsection{Fermionic formula of tau function}

Following Date et al. [23] (see also the work of Kac and van de Leur [25]), we now consider an $N+1$-component and "charged" version of the KP hierarchy in their fermionic formalism. This hierarchy has an $N+1$-tuple $\boldsymbol{t}$ of time variables organized in the vector notation as

$$
\boldsymbol{t}=\left(\boldsymbol{t}_{0}, \boldsymbol{t}_{1}, \ldots, \boldsymbol{t}_{N}\right), \quad \boldsymbol{t}_{\alpha}=\left(t_{\alpha 1}, t_{\alpha 2}, \ldots\right),
$$


and extra "charge" variables

$$
\boldsymbol{s}=\left(s_{0}, s_{1}, \ldots, s_{N}\right), \quad \sum_{\alpha=0}^{N} s_{\alpha}=0 .
$$

The charge variables correspond to the $t_{\alpha 0}$ 's of the universal Whitham hierarchy.

The fermionic formalism is based on an $N+1$-component free fermion system. The one-particle creation-annihilation operators of this system are labeled by $\alpha=0,1, \ldots, N$ as $\psi_{\alpha j}, \psi_{\alpha j}^{*}(j \in \mathbf{Z})$, and obey the anticommuation relations

$$
\left[\psi_{\alpha j}, \psi_{\beta k}^{*}\right]_{+}=\delta_{\alpha \beta} \delta_{j k}, \quad\left[\psi_{\alpha j}, \psi_{\beta k}\right]_{+}=\left[\psi_{\alpha j}^{*}, \psi_{\beta k}^{*}\right]_{+}=0 .
$$

They form an infinite dimensional Clifford algebra. The Fock and dual Fock spaces are generated by the vacuum states $|\mathbf{0}\rangle$ and $\langle\mathbf{0}|$ that satisfy the annihilation conditions

$$
\begin{aligned}
& \left\langle\mathbf{0}\left|\psi_{\alpha j}=0 \quad(j \geq 0), \quad \psi_{\alpha j}\right| \mathbf{0}\right\rangle=0 \quad(j<0), \\
& \left\langle\mathbf{0}\left|\psi_{\alpha j}^{*}=0 \quad(j<0), \quad \psi_{\alpha j}^{*}\right| \mathbf{0}\right\rangle=0 \quad(j \geq 0) .
\end{aligned}
$$

These spaces are decomposed to eigenspaces of the charge operators

$$
H_{\alpha 0}=\sum_{j=-\infty}^{\infty}: \psi_{\alpha j} \psi_{\alpha j}^{*}: \quad \text { (normal ordering) }
$$

each eigenspace being labeled by the charge vector $\boldsymbol{s}=\left(s_{0}, s_{1}, \ldots, s_{N}\right)$ mentioned above. We can choose a ground state $|\boldsymbol{s}\rangle$ and its dual $\langle\boldsymbol{s}|$ in the charge $\boldsymbol{s}$ sector as

$$
\left\langle\boldsymbol{s}\left|=\left\langle\mathbf{0}\left|\Phi_{0 s_{0}} \Phi_{1 s_{1}} \cdots \Phi_{N s_{N}}, \quad\right| \boldsymbol{s}\right\rangle=\Phi_{N s_{N}}^{*} \cdots \Phi_{1 s_{1}}^{*} \Phi_{0 s_{0}}^{*}\right| \mathbf{0}\right\rangle,
$$

where

$$
\begin{aligned}
& \Phi_{\alpha s_{\alpha}}= \begin{cases}\psi_{\alpha, 0}^{*} \cdots \psi_{\alpha, s-1}^{*} & (s>0), \\
\psi_{\alpha,-1} \cdots \psi_{\alpha, s} & (s<0),\end{cases} \\
& \Phi_{\alpha s_{\alpha}}^{*}= \begin{cases}\psi_{\alpha, s-1} \cdots \psi_{\alpha, 0} & (s>0), \\
\psi_{\alpha, s}^{*} \cdots \psi_{\alpha,-1}^{*} & (s<0) .\end{cases}
\end{aligned}
$$

We then have the relation

$$
\langle\boldsymbol{s}| \psi_{\alpha, s_{\alpha}-1}=\epsilon_{\alpha}(\boldsymbol{s})\left\langle\boldsymbol{s}-\boldsymbol{e}_{\alpha}\right|, \quad\langle\boldsymbol{s}| \psi_{\alpha, s_{\alpha}-1}^{*}=\epsilon_{\alpha}(\boldsymbol{s})\left\langle\boldsymbol{s}+\boldsymbol{e}_{\alpha}\right|
$$


and a similar relation for $|\boldsymbol{s}\rangle$, where $\boldsymbol{e}_{\alpha}$ denotes the unit vector

$$
\boldsymbol{e}_{\alpha}=(\ldots, 0,1,0, \ldots) \quad(1 \text { in the } \alpha \text {-th component })
$$

and $\epsilon_{\alpha}(s)$ the sign factor

$$
\epsilon_{\alpha}(s)=(-1)^{s_{\alpha+1}+\cdots+s_{N}} .
$$

The $\tau$-function $\tau(\boldsymbol{s}, \boldsymbol{t})$ of the $N+1$-component KP hierarchy is given by the expectation value

$$
\tau(\boldsymbol{s}, \boldsymbol{t})=\left\langle\boldsymbol{s}\left|e^{H(\boldsymbol{t})} g\right| \mathbf{0}\right\rangle
$$

of a product of two elements $e^{H(\boldsymbol{t})}$ and $g$ of the Clifford groups (i.e., the group of invertible elements of the Clifford algebra whose adjoint action generates a linear transformation on the linear span of one-particle creation-annihilation operators). $H(\boldsymbol{t})$ is the linear combination

$$
H(\boldsymbol{t})=\sum_{\alpha=0}^{N} \sum_{n=1}^{\infty} t_{\alpha n} H_{\alpha n}
$$

of part of the generators

$$
H_{\alpha n}=\sum_{j=-\infty}^{\infty}: \psi_{\alpha, j} \psi_{\alpha, j+n}^{*}:
$$

of an $N+1$-component Heisenberg algebra. $g$ is a general element of the Clifford group whose adjoint action on the linear span of one-particle operators does not mix $\psi_{\alpha j}$ 's and $\psi_{\alpha j}^{*}$ 's:

$$
g \psi_{\beta k} g^{-1}=\sum_{\alpha=0}^{N} \sum_{j=-\infty}^{\infty} a_{\alpha \beta j k} \psi_{\alpha j}, \quad g \psi_{\beta k}^{*} g^{-1}=\sum_{\alpha=0}^{N} \sum_{j=-\infty}^{\infty} \tilde{a}_{\alpha \beta j k} \psi_{\alpha j}^{*}
$$

A typical case is the exponentiated fermion bilinear form

$$
g=\exp \left(\sum_{\alpha, \beta=0}^{N} \sum_{j, k=-\infty}^{\infty} a_{\alpha \beta j k}: \psi_{\alpha j} \psi_{\beta k}^{*}:\right) .
$$

Note that $e^{H(\boldsymbol{t})}$, too, is an operator of this type.

We have two important consequences of this setting. Firstly, the expectation value of (46) vanishes unless $s_{0}+s_{1}+\cdots+s_{N}=0$. Secondly, the 
infinite matrices of the coefficients $a_{\alpha \beta j k}$ and $\tilde{a}_{\alpha \beta j k}$ turn out to be contragradient to each other (i.e., equal to the transposed inverse). This implies that the operator bilinear identity

$$
\sum_{\gamma=0}^{N} \sum_{j=-\infty}^{\infty} \psi_{\gamma j} g \otimes \psi_{\gamma j}^{*} g=\sum_{\gamma=0}^{N} \sum_{j=-\infty}^{\infty} g \psi_{\gamma j} \otimes g \psi_{\gamma j}^{*}
$$

holds, and this identity leads to bilinear equations for the $\tau$-function.

\subsection{Bilinear equations of tau function}

Let us introduce the free fermion fields

$$
\psi_{\alpha}(z)=\sum_{j=-\infty}^{\infty} \psi_{\alpha j} z^{j}, \quad \psi_{\alpha}^{*}(z)=\sum_{j=-\infty}^{\infty} \psi_{\alpha j}^{*} z^{-j-1} .
$$

and rewrite the operator identity (49) to an integral form as

$$
\sum_{\gamma=0}^{N} \oint \frac{d z}{2 \pi i} \psi_{\gamma}(z) g \otimes \psi_{\gamma}^{*}(z) g=\sum_{\gamma=0}^{N} \oint \frac{d z}{2 \pi i} g \psi_{\gamma}(z) \otimes g \psi_{\gamma}^{*}(z) .
$$

The contour integral is understood to be an integral along the circle $|z|=R$ with sufficiently large radius $R$ :

$$
\oint \frac{d z}{2 \pi i} z^{n}=\delta_{n,-1}
$$

We now apply both hand side of this operator identity to $|\mathbf{0}\rangle \otimes|\mathbf{0}\rangle$. A clue is the identity

$$
\psi_{\gamma j}|\mathbf{0}\rangle \otimes \psi_{\gamma j}^{*}|\mathbf{0}\rangle=0
$$

which holds for all $j \in \mathbf{Z}$ because either $\psi_{\gamma j}$ or $\psi_{\gamma j}^{*}$ annihilates $|\mathbf{0}\rangle$. This identity of states in the Fock space implies that

$$
\sum_{\gamma=0}^{N} \oint \frac{d z}{2 \pi i} g \psi_{\gamma}(z)|\mathbf{0}\rangle \otimes g \psi_{\gamma}^{*}(z)|\mathbf{0}\rangle=\sum_{\gamma=0}^{N} \sum_{j=-\infty}^{\infty} g \psi_{\gamma j}|\mathbf{0}\rangle \otimes g \psi_{\gamma j}^{*}|\mathbf{0}\rangle=0 .
$$

Thus we are left with the identity

$$
\sum_{\gamma=0}^{N} \oint \frac{d z}{2 \pi i} \psi_{\gamma}(z) g|\mathbf{0}\rangle \otimes \psi_{\gamma}^{*}(z) g|\mathbf{0}\rangle=0
$$


We now apply $e^{H\left(\boldsymbol{t}^{\prime}\right)} \otimes e^{H(\boldsymbol{t})}$ to the left hand side of the last identity and make the inner product with $\left\langle\boldsymbol{s}^{\prime}+\boldsymbol{e}_{\alpha}\right| \otimes\left\langle\boldsymbol{s}-\boldsymbol{e}_{\beta}\right|$, where $\boldsymbol{t}, \boldsymbol{t}^{\prime}, \boldsymbol{s}, \boldsymbol{s}^{\prime}, \alpha$ and $\beta$ are arbitrary. This yields the equation

$$
\sum_{\gamma=0}^{N} \oint \frac{d z}{2 \pi i}\left\langle\boldsymbol{s}^{\prime}+\boldsymbol{e}_{\alpha}\left|e^{H\left(\boldsymbol{t}^{\prime}\right)} \psi_{\gamma}(z) g\right| \mathbf{0}\right\rangle\left\langle\boldsymbol{s}-\boldsymbol{e}_{\beta}\left|e^{H(\boldsymbol{t})} \psi_{\gamma}^{*}(z) g\right| \mathbf{0}\right\rangle=0
$$

of expectation values. By a multi-component analogue of the well known bosonization formula, the expectation values in the integral can be expressed in terms of the tau function as

$$
\begin{aligned}
\left\langle\boldsymbol{s}^{\prime}+\boldsymbol{e}_{\alpha}\left|e^{H\left(\boldsymbol{t}^{\prime}\right)} \psi_{\gamma}(z) g\right| \mathbf{0}\right\rangle & =\epsilon_{\alpha \gamma}\left(\boldsymbol{s}^{\prime}\right) z^{s_{\gamma}^{\prime}+\delta_{\alpha \gamma}-1} e^{\xi\left(\boldsymbol{t}_{\gamma}^{\prime}, z\right)} e^{-D_{\gamma}(z)} \tau\left(\boldsymbol{s}^{\prime}+\boldsymbol{e}_{\alpha}-\boldsymbol{e}_{\gamma}, \boldsymbol{t}^{\prime}\right), \\
\left\langle\boldsymbol{s}+\boldsymbol{e}_{\beta}\left|e^{H(\boldsymbol{t})} \psi_{\gamma}^{*}(z) g\right| \mathbf{0}\right\rangle & =\epsilon_{\beta \gamma}(\boldsymbol{s}) z^{-s_{\gamma}+\delta_{\beta \gamma}-1} e^{-\xi\left(\boldsymbol{t}_{\gamma}, z\right)} e^{D_{\gamma}(z)} \tau\left(\boldsymbol{s}-\boldsymbol{e}_{\beta}+\boldsymbol{e}_{\gamma}, \boldsymbol{t}\right),
\end{aligned}
$$

where $D_{\gamma}(z)$ is the differential operator that has been used in the formula (26) of the $S$-functions, and the other notations are those commonly used for the (multi-component) KP hierarchy, namely,

$$
\epsilon_{\beta \gamma}(\boldsymbol{s})=\left\{\begin{array}{ll}
(-1)^{s_{\beta+1}+\cdots+s_{\gamma}} & (\beta \leq \gamma), \\
-(-1)^{s_{\gamma+1}+\cdots+s_{\beta}} & (\beta>\gamma),
\end{array} \quad \xi\left(\boldsymbol{t}_{\gamma}, z\right)=\sum_{n=1}^{\infty} t_{\gamma n} z^{n} .\right.
$$

Thus the foregoing bilinear equation of expectation values turns into the bilinear equation

$$
\begin{aligned}
& \sum_{\gamma=0}^{N} \epsilon_{\alpha \gamma}\left(\boldsymbol{s}^{\prime}\right) \epsilon_{\beta \gamma}(\boldsymbol{s}) \oint \frac{d z}{2 \pi i} z^{s_{\gamma}^{\prime}-s_{\gamma}+\delta_{\alpha \gamma}+\delta_{\beta \gamma}-2} e^{\xi\left(\boldsymbol{t}_{\gamma}^{\prime}-\boldsymbol{t}_{\gamma}, z\right)} \times \\
& \times\left(e^{-D_{\gamma}(z)} \tau\right)\left(\boldsymbol{s}^{\prime}+\boldsymbol{e}_{\alpha}-\boldsymbol{e}_{\gamma}, \boldsymbol{t}^{\prime}\right)\left(e^{D_{\gamma}(z)} \tau\right)\left(\boldsymbol{s}-\boldsymbol{e}_{\beta}+\boldsymbol{e}_{\gamma}, \boldsymbol{t}\right)=0
\end{aligned}
$$

of the $\tau$-function, which holds for arbitrary value of $\boldsymbol{t}^{\prime}, \boldsymbol{t}, \boldsymbol{s}^{\prime}, \boldsymbol{s}$ and for $\alpha, \beta=$ $0,1, \ldots, N$. By the standard procedure, this bilinear equation can be converted to an infinite number of Hirota equations.

The subsequent consideration is focused on the case where $\alpha=\beta=0$, which actually covers the general case by shifting $s$ and $\boldsymbol{s}^{\prime}$. For convenience, we separate the term of $\gamma=0$ from the sum over $\gamma=0,1, \ldots, N$ and move the sign factors $\epsilon_{00}\left(\boldsymbol{s}^{\prime}\right)$ and $\epsilon_{00}(\boldsymbol{s})$ to the other terms. $\epsilon_{0 \gamma}\left(\boldsymbol{s}^{\prime}\right)$ and $\epsilon_{0 \gamma}(\boldsymbol{s})$ of the latter are thereby replaced by

$$
\tilde{\epsilon}_{\gamma}\left(\boldsymbol{s}^{\prime}\right)=\frac{\epsilon_{0 \gamma}\left(\boldsymbol{s}^{\prime}\right)}{\epsilon_{00}\left(\boldsymbol{s}^{\prime}\right)}=(-1)^{s_{1}^{\prime}+\cdots+s_{\gamma}^{\prime}}, \quad \tilde{\epsilon}_{\gamma}(\boldsymbol{s})=\frac{\epsilon_{0 \gamma}(\boldsymbol{s})}{\epsilon_{00}(\boldsymbol{s})}=(-1)^{s_{1}+\cdots+s_{\gamma}} .
$$


Bilinear equation (53) for $\alpha=\beta=0$ thus reads

$$
\begin{aligned}
& \oint \frac{d z}{2 \pi i} z^{s_{0}^{\prime}-s_{0}} e^{\xi\left(\boldsymbol{t}_{0}^{\prime}-\boldsymbol{t}_{0}, z\right)}\left(e^{-D_{0}(z)} \tau\right)\left(\boldsymbol{s}^{\prime}, \boldsymbol{t}^{\prime}\right)\left(e^{D_{0}(z)} \tau\right)(\boldsymbol{s}, \boldsymbol{t}) \\
+ & \sum_{\gamma=1}^{N} \tilde{\epsilon}_{\gamma}\left(\boldsymbol{s}^{\prime}\right) \tilde{\epsilon}_{\gamma}(\boldsymbol{s}) \oint \frac{d z}{2 \pi i} z^{s_{\gamma}^{\prime}-s_{\gamma}-2} e^{\xi\left(\boldsymbol{t}_{\gamma}^{\prime}-\boldsymbol{t}_{\gamma}, z\right)} \times \\
& \times\left(e^{-D_{\gamma}(z)} \tau\right)\left(\boldsymbol{s}^{\prime}+\boldsymbol{e}_{0}-\boldsymbol{e}_{\gamma}, \boldsymbol{t}^{\prime}\right)\left(e^{D_{\gamma}(z)} \tau\right)\left(\boldsymbol{s}-\boldsymbol{e}_{0}+\boldsymbol{e}_{\gamma}, \boldsymbol{t}\right)=0 .
\end{aligned}
$$

We shall show later that the sign factor $\tilde{\epsilon}_{\gamma}(\boldsymbol{e})$ is related to the factor proportional to $\log (-1)$ in the defining equations (25) of the $F$-function.

\section{Dispersionless Hirota equations as dipser- sionless limit of differential Fay identities}

\subsection{How to derive differential Fay identity}

Let us recall the differential Fay identity for the $\tau$-function $\tau(\boldsymbol{t}), \boldsymbol{t}=\left(t_{1}, t_{2}, \ldots\right)$, of the KP hierarchy [3]. This identity is usually derived by taking a limit of the three-term Fay identity

$$
\begin{aligned}
& \left(z_{0}-z_{1}\right)\left(z_{2}-z_{3}\right) \tau\left(\boldsymbol{t}+\left[z_{0}\right]+\left[z_{1}\right]\right) \tau\left(\boldsymbol{t}+\left[z_{2}\right]+\left[z_{3}\right]\right) \\
& +\left(\text { cyclic permutations of } z_{1}, z_{2}, z_{3}\right)=0,
\end{aligned}
$$

where $[z]$ denotes the shift vector

$$
[z]=\left(z, z^{2} / 2, \ldots, z^{n} / n, \ldots\right)
$$

in the time variables.

Actually, there is an alternative method to derive the differential Fay identity as follows. This alternative method consists of the following steps. The first step is to differentiate the bilinear equation

$$
\oint \frac{d z}{2 \pi i} e^{\xi\left(\boldsymbol{t}^{\prime}-\boldsymbol{t}, z\right)}\left(e^{-D(z)} \tau\right)\left(\boldsymbol{t}^{\prime}\right)\left(e^{D(z)} \tau\right)(\boldsymbol{t})=0
$$

by $t_{1}^{\prime}$. This yields an equation of the form

$$
\oint \frac{d z}{2 \pi i} e^{\xi\left(\boldsymbol{t}^{\prime}-\boldsymbol{t}, z\right)}\left(z\left(e^{-D(z)} \tau\right)\left(\boldsymbol{t}^{\prime}\right)+\left(\partial_{1} e^{-D(z)} \tau\right)\left(\boldsymbol{t}^{\prime}\right)\right) \tau\left(\boldsymbol{t}+\left[z^{-1}\right]\right)=0,
$$

where $\partial_{1}$ denotes the differential operator

$$
\partial_{1}=\partial / \partial t_{1}
$$


The next step is to specialize $\boldsymbol{t}^{\prime}$ as

$$
\boldsymbol{t}^{\prime}=\boldsymbol{t}+\left[\lambda^{-1}\right]+\left[\mu^{-1}\right]
$$

where $\lambda$ and $\mu$ are arbitrary constants that sit on the far side of the contour $|z|=R$ of the integral, namely,

$$
|\lambda|>R, \quad|\mu|>R
$$

The exponential factor $e^{\xi\left(\boldsymbol{t}^{\prime}-\boldsymbol{t}, z\right)}$ thereby reduces to

$$
e^{\xi\left(\boldsymbol{t}^{\prime}-\boldsymbol{t}, z\right)}=\left(1-\frac{z}{\lambda}\right)^{-1}\left(1-\frac{z}{\mu}\right)^{-1}=\frac{\lambda \mu}{(z-\lambda)(z-\mu)}
$$

and the foregoing bilinear equation now takes such a form as

$$
\begin{aligned}
\oint \frac{d z}{2 \pi i} \frac{\lambda \mu}{(z-\lambda)(z-\mu)}\left(z \tau\left(\boldsymbol{t}+\left[\lambda^{-1}\right]+\left[\mu^{-1}\right]-\left[z^{-1}\right]\right)+\right. \\
\left.+\left(\partial_{1} \tau\right)\left(\boldsymbol{t}+\left[\lambda^{-1}\right]+\left[\mu^{-1}\right]-\left[z^{-1}\right]\right)\right) \tau\left(\boldsymbol{t}+\left[z^{-1}\right]\right)=0 .
\end{aligned}
$$

Assuming, as usual, that the factors including the $\tau$-function are holomorphic functions of $z$ on the far side of the contour, one can deform the contour to a union of circles that encircle the poles at $z=\lambda, \mu, \infty$. The contour integrals along those circles are given by residues. Collecting these pieces, one eventually obtains the equation

$$
\begin{aligned}
& (\lambda-\mu) \tau\left(\boldsymbol{t}+\left[\lambda^{-1}\right]+\left[\mu^{-1}\right]\right) \tau(\boldsymbol{t})-\tau\left(\boldsymbol{t}+\left[\lambda^{-1}\right]\right) \tau\left(\boldsymbol{t}+\left[\mu^{-1}\right]\right) \\
& +\left(\partial_{1} \tau\right)\left(\boldsymbol{t}+\left[\lambda^{-1}\right]\right) \tau\left(\boldsymbol{t}+\left[\mu^{-1}\right]\right)-\left(\partial_{1} \tau\right)\left(\boldsymbol{t}+\left[\mu^{-1}\right]\right) \tau\left(\boldsymbol{t}+\left[\lambda^{-1}\right]\right)=0,
\end{aligned}
$$

which is essentially the differential Fay identity.

This equation can be cast into a form that is more suited for considering the dispersionless limit. Let us divide both hand side of this equation by $(\lambda-\mu) \tau\left(\boldsymbol{t}+\left[\lambda^{-1}\right]\right) \tau\left(\boldsymbol{t}+\left[\mu^{-1}\right]\right)$. This leads to an equation of the form

$$
\frac{\tau\left(\boldsymbol{t}+\left[\lambda^{-1}\right]+\left[\mu^{-1}\right]\right) \tau(\boldsymbol{t})}{\tau\left(\boldsymbol{t}+\left[\lambda^{-1}\right]\right) \tau\left(\boldsymbol{t}+\left[\mu^{-1}\right]\right)}=1-\frac{\partial_{1}\left(\log \tau\left(\boldsymbol{t}+\left[\lambda^{-1}\right]\right)-\log \tau\left(\boldsymbol{t}+\left[\mu^{-1}\right]\right)\right)}{\lambda-\mu}
$$

Using the differential operator

$$
D(z)=\sum_{n=1}^{\infty} \frac{z^{-n}}{n} \partial_{n}, \quad \partial_{n}=\partial / \partial t_{n}
$$

we can rewrite the last equation as

$$
\exp \left(\left(e^{D(\lambda)}-1\right)\left(e^{D(\mu)}-1\right) \log \tau(\boldsymbol{t})\right)=1-\frac{\partial_{1}\left(e^{D(\lambda)}-e^{D(\mu)}\right) \log \tau(\boldsymbol{t})}{\lambda-\mu} .
$$

This shows a fully "dispersive" form of the dispersionless Hirota equation

$$
e^{D(\lambda) D(\mu) F}=1-\frac{\partial_{1}(D(\lambda)-D(\mu)) F}{\lambda-\mu} .
$$




\subsection{Multi-component analogue of differential Fay iden- tity}

The foregoing method turns out to work for the bilinear equations (154) of the multi-component case as well. We thus obtain a multi-component analogue, (61) -(64), of the differential Fay identity as follows.

Theorem 2. The $\tau$-function satisfies the multi-component differential Fay identities

$$
\begin{aligned}
& \exp \left(\left(e^{D_{0}(\lambda)}-1\right)\left(e^{D_{0}(\mu)}-1\right) \log \tau(\boldsymbol{s}, \boldsymbol{t})\right) \\
& =1-\frac{\partial_{01}\left(e^{D_{0}(\lambda)}-e^{D_{0}(\mu)}\right) \log \tau(\boldsymbol{s}, \boldsymbol{t})}{\lambda-\mu}, \\
& \begin{aligned}
& \lambda \exp \left(\left(e^{D_{0}(\lambda)}-1\right)\right.\left.\left(e^{\partial_{\alpha 0}+D_{\alpha}(\mu)}-1\right) \log \tau(\boldsymbol{s}, \boldsymbol{t})\right) \\
&= \lambda-\partial_{01}\left(e^{D_{0}(\lambda)}-e^{\partial_{\alpha 0}+D_{\alpha}(\mu)}\right) \log \tau(\boldsymbol{s}, \boldsymbol{t}), \\
& \exp \left(\left(e^{\partial_{\alpha 0}+D_{\alpha}(\lambda)}-1\right)\left(e^{\partial_{\alpha 0}+D_{\alpha}(\mu)}-1\right) \log \tau(\boldsymbol{s}, \boldsymbol{t})\right) \lambda-\mu \\
&=-\frac{\lambda \mu \partial_{01}\left(e^{\partial_{\alpha 0}+D_{\alpha}(\lambda)}-e^{\partial_{\alpha 0}+D_{\alpha}(\mu)}\right) \log \tau(\boldsymbol{s}, \boldsymbol{t})}{\lambda-\mu}, \\
& \epsilon_{\alpha \beta} \exp \left(\left(e^{\partial_{\alpha 0}+D_{\alpha}(\lambda)}-1\right)\left(e^{\partial_{\beta 0}+D_{\beta}(\mu)}-1\right) \log \tau(\boldsymbol{s}, \boldsymbol{t})\right) \\
&=-\partial_{01}\left(e^{\partial_{\alpha 0}+D_{\alpha}(\lambda)}-e^{\partial_{\beta 0}+D_{\beta}(\mu)}\right) \log \tau(\boldsymbol{s}, \boldsymbol{t}),
\end{aligned}
\end{aligned}
$$

where $e^{\partial_{\alpha 0}}$ is understood to be the shift operator in the $\boldsymbol{s}$ variables, i.e.,

$$
e^{\partial_{\alpha 0}} f(\boldsymbol{s})=f\left(\boldsymbol{s}-\boldsymbol{e}_{0}+\boldsymbol{e}_{\alpha}\right) .
$$

Proof. To derive (61), we differentiate (54) by $t_{01}^{\prime}$ and put

$$
\boldsymbol{t}_{0}^{\prime}=\boldsymbol{t}_{0}+\left[\lambda^{-1}\right]+\left[\mu^{-1}\right], \quad \boldsymbol{t}_{\alpha}^{\prime}=\boldsymbol{t}_{\alpha} \quad(\alpha=1, \ldots, N), \quad \boldsymbol{s}^{\prime}=\boldsymbol{s} .
$$

The differentiated bilinear equation thereby simplifies as

$$
\begin{aligned}
& \oint \frac{d z}{2 \pi i} \frac{\lambda \mu}{(z-\lambda)(z-\mu)}\left(z \tau\left(\boldsymbol{s}, \boldsymbol{t}+\left[\lambda^{-1}\right]_{0}+\left[\mu^{-1}\right]_{0}-\left[z^{-1}\right]_{0}\right)+\right. \\
& \left.\quad+\left(\partial_{01} \tau\right)\left(\boldsymbol{s}, \boldsymbol{t}+\left[\lambda^{-1}\right]_{0}+\left[\mu^{-1}\right]_{0}-\left[z^{-1}\right]_{0}\right)\right) \tau\left(\boldsymbol{s}, \boldsymbol{t}+\left[z^{-1}\right]_{0}\right)=0,
\end{aligned}
$$

where $[z]_{\alpha}$ now stands for the shift vector

$$
[z]_{\alpha}=(\ldots, \mathbf{0},[z], \mathbf{0}, \ldots) \quad([z] \text { in the } \alpha \text {-th component })
$$

in the multi-component time variables. This is substantially the same bilinear equation as mentioned above for the case of the usual KP hierarchy. We thus 
obtain the equation

$$
\begin{aligned}
& \frac{\tau\left(\boldsymbol{s}, \boldsymbol{t}+\left[\lambda^{-1}\right]_{0}+\left[\mu^{-1}\right]_{0}\right) \tau(\boldsymbol{s}, \boldsymbol{t})}{\tau\left(\boldsymbol{s}, \boldsymbol{t}+\left[\lambda^{-1}\right]_{0}\right) \tau\left(\boldsymbol{s}, \boldsymbol{t}+\left[\mu^{-1}\right]_{0}\right)} \\
& =1-\frac{\partial_{01}\left(\log \tau\left(\boldsymbol{s}, \boldsymbol{t}+\left[\lambda^{-1}\right]_{0}\right)-\log \tau\left(\boldsymbol{s}, \boldsymbol{t}+\left[\mu^{-1}\right]_{0}\right)\right)}{\lambda-\mu}
\end{aligned}
$$

which we can readily rewrite to (61).

To derive (62), we differentiate (154) by $t_{01}^{\prime}$ and put

$$
\begin{array}{r}
\boldsymbol{t}_{0}^{\prime}=\boldsymbol{t}_{0}+\left[\lambda^{-1}\right], \quad \boldsymbol{t}_{\alpha}^{\prime}=\boldsymbol{t}_{\alpha}+\left[\mu^{-1}\right], \quad \boldsymbol{t}_{\beta}^{\prime}=\boldsymbol{t}_{\beta} \quad(\beta \neq \alpha), \\
s_{0}^{\prime}=s_{0}-1, \quad s_{\alpha}^{\prime}=s_{\alpha}+1, \quad s_{\beta}^{\prime}=s_{\beta} \quad(\beta \neq \alpha) .
\end{array}
$$

The differentiated bilinear equation takes such a form as

$$
\begin{aligned}
-\oint & \frac{d z}{2 \pi i} \frac{1}{z} \frac{-\lambda}{z-\lambda}\left(z \tau\left(\boldsymbol{s}-\boldsymbol{e}_{0}+\boldsymbol{e}_{\alpha}, \boldsymbol{t}+\left[\lambda^{-1}\right]_{0}-\left[z^{-1}\right]_{0}+\left[\mu^{-1}\right]_{\alpha}\right)+\right. \\
& \left.+\left(\partial_{01} \tau\right)\left(\boldsymbol{s}-\boldsymbol{e}_{0}+\boldsymbol{e}_{\alpha}, \boldsymbol{t}+\left[\lambda^{-1}\right]_{0}-\left[z^{-1}\right]_{0}+\left[\mu^{-1}\right]_{\alpha}\right)\right) \times \\
& \times \tau\left(\boldsymbol{s}, \boldsymbol{t}+\left[z^{-1}\right]_{0}\right) \\
+\oint & \frac{d z}{2 \pi i} \frac{1}{z} \frac{-\mu}{z-\mu}\left(\partial_{01} \tau\right)\left(\boldsymbol{s}, \boldsymbol{t}+\left[\lambda^{-1}\right]_{0}+\left[\mu^{-1}\right]_{\alpha}-\left[z^{-1}\right]_{\alpha}\right) \times \\
\quad \times & \tau\left(\boldsymbol{s}-\boldsymbol{e}_{0}+\boldsymbol{e}_{\alpha}, \boldsymbol{t}+\left[z^{-1}\right]_{\alpha}\right)=0 .
\end{aligned}
$$

After calculating the contour integrals, this equation reduces to

$$
\begin{aligned}
& \lambda \tau\left(\boldsymbol{s}-\boldsymbol{e}_{0}+\boldsymbol{e}_{\alpha}, \boldsymbol{t}+\left[\lambda^{-1}\right]_{0}+\left[\mu^{-1}\right]_{\alpha}\right) \tau(\boldsymbol{s}, \boldsymbol{t}) \\
& -\lambda \tau\left(\boldsymbol{s}-\boldsymbol{e}_{0}+\boldsymbol{e}_{\alpha}, \boldsymbol{t}+\left[\mu^{-1}\right]_{\alpha}\right) \tau\left(\boldsymbol{s}, \boldsymbol{t}+\left[\lambda^{-1}\right]_{0}\right) \\
& -\left(\partial_{01} \tau\right)\left(\boldsymbol{s}-\boldsymbol{e}_{0}+\boldsymbol{e}_{\alpha}, \boldsymbol{t}+\left[\mu^{-1}\right]_{\alpha}\right) \tau\left(\boldsymbol{s}, \boldsymbol{t}+\left[\lambda^{-1}\right]_{0}\right) \\
& +\left(\partial_{01} \tau\right)\left(\boldsymbol{s}, \boldsymbol{t}+\left[\lambda^{-1}\right]_{0}\right) \tau\left(\boldsymbol{s}-\boldsymbol{e}_{0}+\boldsymbol{e}_{\alpha}, \boldsymbol{t}+\left[\mu^{-1}\right]_{\alpha}\right)=0
\end{aligned}
$$

which we can further rewrite as

$$
\begin{aligned}
& \lambda \frac{\tau\left(\boldsymbol{s}-\boldsymbol{e}_{0}+\boldsymbol{e}_{\alpha}, \boldsymbol{t}+\left[\lambda^{-1}\right]_{0}+\left[\mu^{-1}\right]_{\alpha}\right) \tau(\boldsymbol{s}, \boldsymbol{t})}{\tau\left(\boldsymbol{s}, \boldsymbol{t}+\left[\lambda^{-1}\right]_{0}\right) \tau\left(\boldsymbol{s}, \boldsymbol{t}+\left[\mu^{-1}\right]_{\alpha}\right)} \\
& =\lambda-\partial_{01}\left(\log \tau\left(\boldsymbol{s}, \boldsymbol{t}+\left[\lambda^{-1}\right]_{0}\right)-\log \tau\left(\boldsymbol{s}-\boldsymbol{e}_{0}+\boldsymbol{e}_{\alpha}, \boldsymbol{t}+\left[\mu^{-1}\right]_{\alpha}\right)\right) .
\end{aligned}
$$

After some more algebra, this equation becomes (62).

To derive (63), we choose

$$
\begin{aligned}
& \boldsymbol{t}_{\alpha}^{\prime}=\boldsymbol{t}_{\alpha}+\left[\lambda^{-1}\right]+\left[\mu^{-1}\right], \quad \boldsymbol{t}_{\beta}^{\prime}=\boldsymbol{t}_{\beta} \quad(\beta \neq \alpha), \\
s_{0}^{\prime}= & s_{0}-2, \quad s_{\alpha}^{\prime}=s_{\alpha}+2, \quad s_{\beta}^{\prime}=s_{\beta} \quad(\beta \neq 0, \alpha) .
\end{aligned}
$$


The differentiated bilinear equation now reads

$$
\begin{aligned}
\oint & \frac{d z}{2 \pi i}\left(z \tau\left(\boldsymbol{s}-2 \boldsymbol{e}_{0}+2 \boldsymbol{e}_{\alpha}, \boldsymbol{t}-\left[z^{-1}\right]_{0}+\left[\lambda^{-1}\right]_{\alpha}+\left[\mu^{-1}\right]_{\alpha}\right)+\right. \\
& \left.+\left(\partial_{01} \tau\right)\left(\boldsymbol{s}-\boldsymbol{e}_{0}+\boldsymbol{e}_{\alpha}, \boldsymbol{t}-\left[z^{-1}\right]_{0}+\left[\lambda^{-1}\right]_{\alpha}+\left[\mu^{-1}\right]_{\alpha}\right)\right) \times \\
& \times \tau\left(\boldsymbol{s}, \boldsymbol{t}+\left[z^{-1}\right]_{0}\right) \\
+\oint & \frac{d z}{2 \pi i} \frac{\lambda \mu}{(z-\lambda)(z-\mu)}\left(\partial_{01} \tau\right)\left(\boldsymbol{s}-\boldsymbol{e}_{0}+\boldsymbol{e}_{\alpha}, \boldsymbol{t}+\left[\lambda^{-1}\right]_{\alpha}+\left[\mu^{-1}\right]_{\alpha}\right) \times \\
& \times \tau\left(\boldsymbol{s}-\boldsymbol{e}_{0}+\boldsymbol{e}_{\alpha}, \boldsymbol{t}+\left[z^{-1}\right]_{0}\right)=0 .
\end{aligned}
$$

The outcome of residue calculus is the equation

$$
\begin{aligned}
& \frac{\tau\left(\boldsymbol{s}-2 \boldsymbol{e}_{0}+2 \boldsymbol{e}_{\alpha}, \boldsymbol{t}+\left[\lambda^{-1}\right]_{\alpha}+\left[\mu^{-1}\right]_{\alpha}\right) \tau(\boldsymbol{s}, \boldsymbol{t})}{\tau\left(\boldsymbol{s}-\boldsymbol{e}_{0}+\boldsymbol{e}_{\alpha}, \boldsymbol{t}+\left[\lambda^{-1}\right]_{\alpha}\right) \tau\left(\boldsymbol{s}-\boldsymbol{e}_{0}+\boldsymbol{e}_{\alpha}, \boldsymbol{t}+\left[\mu^{-1}\right]_{\alpha}\right)}=-\frac{\lambda \mu}{\lambda-\mu} \times \\
& \times \partial_{01}\left(\log \tau\left(\boldsymbol{s}-\boldsymbol{e}_{0}+\boldsymbol{e}_{\alpha}, \boldsymbol{t}+\left[\lambda^{-1}\right]_{\alpha}\right)-\log \tau\left(\boldsymbol{s}-\boldsymbol{e}_{0}+\boldsymbol{e}_{\alpha}, \boldsymbol{t}+\left[\mu^{-1}\right]_{\alpha}\right)\right),
\end{aligned}
$$

which becomes (63) after some algebra.

Lastly, we derive (64). This is achieved by choosing

$$
\begin{aligned}
& \boldsymbol{t}_{\alpha}^{\prime}=\boldsymbol{t}_{\alpha}+\left[\lambda^{-1}\right], \quad \boldsymbol{t}_{\beta}^{\prime}=\boldsymbol{t}_{\beta}+\left[\mu^{-1}\right], \boldsymbol{t}_{\gamma}^{\prime}=\boldsymbol{t}_{\gamma} \quad(\gamma \neq \alpha, \beta), \\
& s_{0}^{\prime}=s_{0}-2, \quad s_{\alpha}^{\prime}=s_{\alpha}+1, \quad s_{\beta}^{\prime}=s_{\beta}+1, \quad s_{\gamma}^{\prime}=s_{\gamma} \quad(\gamma \neq \alpha, \beta),
\end{aligned}
$$

and repeating the same procedure as the previous cases. Unlike those cases, however, we now have to account for the sign factors in (54). The outcome takes a different form for $\alpha<\beta$ and $\alpha>\beta$ as follows:

$$
\begin{aligned}
\oint & \frac{d z}{2 \pi i} \frac{1}{z^{2}}\left(z \tau\left(\boldsymbol{s}-2 \boldsymbol{e}_{0}+\boldsymbol{e}_{\alpha}+\boldsymbol{e}_{\beta}, \boldsymbol{t}-\left[z^{-1}\right]_{0}+\left[\lambda^{-1}\right]_{\alpha}+\left[\mu^{-1}\right]_{\beta}\right)+\right. \\
& \left.+\left(\partial_{01} \tau\right)\left(\boldsymbol{s}-2 \boldsymbol{e}_{0}+\boldsymbol{e}_{\alpha}+\boldsymbol{e}_{\beta}, \boldsymbol{t}-\left[z^{-1}\right]_{0}+\left[\lambda^{-1}\right]_{\alpha}+\left[\mu^{-1}\right]_{\beta}\right)\right) \times \\
& \times \tau\left(\boldsymbol{s}, \boldsymbol{t}+\left[z^{-1}\right]_{0}\right) \\
-\epsilon_{\alpha \beta} \oint & \frac{d z}{2 \pi i} \frac{1}{z} \frac{-\lambda}{z-\lambda}\left(\partial_{01} \tau\right)\left(\boldsymbol{s}-\boldsymbol{e}_{0}+\boldsymbol{e}_{\beta}, \boldsymbol{t}+\left[\lambda^{-1}\right]_{\alpha}-\left[z^{-1}\right]_{\alpha}+\left[\mu^{-1}\right]_{\beta}\right) \times \\
& \times \tau\left(\boldsymbol{s}-\boldsymbol{e}_{0}+\boldsymbol{e}_{\alpha}, \boldsymbol{t}+\left[z^{-1}\right]_{\alpha}\right) \\
+\epsilon_{\alpha \beta} \oint & \frac{d z}{2 \pi i} \frac{1}{z} \frac{-\mu}{z-\mu}\left(\partial_{01} \tau\right)\left(\boldsymbol{e}+\boldsymbol{e}_{\alpha}, \boldsymbol{t}+\left[\lambda^{-1}\right]_{\alpha}+\left[\mu^{-1}\right]_{\beta}-\left[z^{-1}\right]_{\beta}\right) \times \\
& \times \tau\left(\boldsymbol{s}-\boldsymbol{e}_{0}+\boldsymbol{e}_{\beta}, \boldsymbol{t}+\left[z^{-1}\right]_{\beta}\right)=0,
\end{aligned}
$$

where $\epsilon_{\alpha \beta}$ stands for the same sign factor as in (41). This equation reduces to

$$
\begin{aligned}
& \epsilon_{\alpha \beta} \frac{\tau\left(\boldsymbol{s}-2 \boldsymbol{e}_{0}+\boldsymbol{e}_{\alpha}+\boldsymbol{e}_{\beta}, \boldsymbol{t}+\left[\lambda^{-1}\right]_{\alpha}+\left[\mu^{-1}\right]_{\beta}\right) \tau(\boldsymbol{s}, \boldsymbol{t})}{\tau\left(\boldsymbol{s}-\boldsymbol{e}_{0}+\boldsymbol{e}_{\alpha}, \boldsymbol{t}+\left[\lambda^{-1}\right]_{\alpha}\right) \tau\left(\boldsymbol{s}-\boldsymbol{e}_{0}+\boldsymbol{e}_{\beta}, \boldsymbol{t}+\left[\mu^{-1}\right]_{\beta}\right)} \\
& =-\partial_{01}\left(\log \tau\left(\boldsymbol{s}-\boldsymbol{e}_{0}+\boldsymbol{e}_{\alpha}, \boldsymbol{t}+\left[\lambda^{-1}\right]_{\alpha}\right)-\log \tau\left(\boldsymbol{s}-\boldsymbol{e}_{0}+\boldsymbol{e}_{\beta}, \boldsymbol{t}+\left[\mu^{-1}\right]_{\beta}\right)\right),
\end{aligned}
$$

and we eventually obtain (64). 


\subsection{Dispersionless limit}

Dispersionless limit is realized as quasi-classical limit. Namely, we allow the $\tau$-function to depend on the "Planck constant" $\hbar$ as well, i.e., $\tau=\tau(\hbar, \boldsymbol{s}, \boldsymbol{t})$, and assume that the rescaled $\tau$-function

$$
\tau_{\hbar}(\boldsymbol{s}, \boldsymbol{t})=\tau\left(\hbar, \hbar^{-1} \boldsymbol{s}, \hbar^{-1} \boldsymbol{t}\right)
$$

behaves as

$$
\tau_{\hbar}(\boldsymbol{s}, \boldsymbol{t})=\exp \left(\hbar^{-2} F(\boldsymbol{s}, \boldsymbol{t})+O\left(\hbar^{-1}\right)\right)
$$

in the classical limit $\hbar \rightarrow 0$.

The rescaled $\tau$-function satisfies (61)-(64) with the operators $e^{D_{0}(\lambda)}$ etc. being also recalled:

$$
\begin{gathered}
\exp \left(\left(e^{\hbar D_{0}(\lambda)}-1\right)\left(e^{\hbar D_{0}(\mu)}-1\right) \log \tau_{\hbar}(\boldsymbol{s}, \boldsymbol{t})\right) \\
=1-\frac{\hbar \partial_{01}\left(e^{\hbar D_{0}(\lambda)}-e^{\hbar D_{0}(\mu)}\right) \log \tau_{\hbar}(\boldsymbol{s}, \boldsymbol{t})}{\lambda-\mu}, \\
\begin{aligned}
& \lambda \exp \left(\left(e^{\hbar D_{0}(\lambda)-1}-\right.\right.\left.1)\left(e^{\partial_{\hbar \alpha}+\hbar D_{\alpha}(\mu)}-1\right) \log \tau_{\hbar}(\boldsymbol{s}, \boldsymbol{t})\right) \\
&=\lambda-\hbar \partial_{01}\left(e^{D_{0}(\lambda)}-e^{\hbar \partial_{\alpha 0}+\hbar D_{\alpha}(\mu)}\right) \log \tau(\boldsymbol{s}, \boldsymbol{t}), \\
& \exp \left(\left(e^{\hbar \partial_{\alpha 0}+\hbar D_{\alpha}(\lambda)}-\right.\right.\left.1)\left(e^{\hbar \partial_{\alpha 0}+\hbar D_{\alpha}(\mu)}-1\right) \log \tau_{\hbar}(\boldsymbol{s}, \boldsymbol{t})\right) \\
&=-\frac{\lambda \mu \hbar \partial_{01}\left(e^{\hbar \partial_{\alpha 0}+\hbar D_{\alpha}(\lambda)}-e^{\hbar \partial_{\alpha 0}+\hbar D_{\alpha}(\mu)}\right) \log \tau_{\hbar}(\boldsymbol{s}, \boldsymbol{t})}{\lambda-\mu}, \\
& \epsilon_{\alpha \beta} \exp \left(\left(e^{\hbar \partial_{\alpha 0}+\hbar D_{\alpha}(\lambda)}-1\right)\left(e^{\hbar \partial_{\beta 0}+\hbar D_{\beta}(\mu)}-1\right) \log \tau_{\hbar}(\boldsymbol{s}, \boldsymbol{t})\right) \\
&=-\hbar \partial_{01}\left(e^{\hbar \partial_{\alpha 0}+\hbar D_{\alpha}(\lambda)}-e^{\hbar \partial_{\beta 0}+\hbar D_{\beta}(\mu)}\right) \log \tau_{\hbar}(\boldsymbol{s}, \boldsymbol{t}) .
\end{aligned}
\end{gathered}
$$

If we substitute the aforementioned quasi-classical ansatz of the rescaled $\tau$ function and take the limit as $\hbar \rightarrow 0$, we end up with the dipsersionless Hirota equations (38)-(41) for the $F$-function. In other words, the multi-component analogues (61) - (64) of the differential Fay identity are "dispersive" counterparts of the dispersionless Hirota equations.

\section{Differential Fay identities as generating func- tional form of auxiliary linear equations}

\subsection{How to derive auxiliary linear equations}

It is known that the differential Fay identity is actually equivalent to the KP hierarchy itself. This is a consequence of the fact that the auxiliary 
linear equations of the KP hierarchy can be derived from the differential Fay identity. Let us briefly review this fact [2, Appendix C] (see also Teo's paper [16] for an analogous result and its proof for the Toda hierarchy).

To derive auxiliary linear equations, we write the differential Fay identity as

$$
\frac{\tau\left(\boldsymbol{t}+\left[\lambda^{-1}\right]+\left[\mu^{-1}\right]\right) \tau(\boldsymbol{t})}{\tau\left(\boldsymbol{t}+\left[\lambda^{-1}\right]\right) \tau\left(\boldsymbol{t}+\left[\mu^{-1}\right]\right)}=1+\frac{1}{\lambda-\mu} \partial_{1} \log \frac{\tau\left(\boldsymbol{t}+\left[\mu^{-1}\right]\right)}{\tau\left(\boldsymbol{t}+\left[\lambda^{-1}\right]\right)}
$$

and shift the variables as $\boldsymbol{t} \rightarrow \boldsymbol{t}-\left[\lambda^{-1}\right]-\left[\mu^{-1}\right]$. This leads to another form

$$
\frac{\tau\left(\boldsymbol{t}-\left[\lambda^{-1}\right]-\left[\mu^{-1}\right]\right) \tau(\boldsymbol{t})}{\tau\left(\boldsymbol{t}-\left[\lambda^{-1}\right]\right) \tau\left(\boldsymbol{t}-\left[\mu^{-1}\right]\right)}=1+\frac{1}{\lambda-\mu} \partial_{1} \log \frac{\tau\left(\boldsymbol{t}-\left[\lambda^{-1}\right]\right)}{\tau\left(\boldsymbol{t}-\left[\mu^{-1}\right]\right)}
$$

of the differential Fay identity. We can rewrite it as

$$
e^{-D(\lambda)} \frac{\tau\left(\boldsymbol{t}-\left[\mu^{-1}\right]\right)}{\tau(\boldsymbol{t})}=\frac{\tau\left(\boldsymbol{t}-\left[\mu^{-1}\right]\right)}{\tau(\boldsymbol{t})}\left(1+\frac{1}{\lambda-\mu} \partial_{1} \log \frac{\tau\left(\boldsymbol{t}-\left[\lambda^{-1}\right]\right)}{\tau\left(\boldsymbol{t}-\left[\mu^{-1}\right]\right)}\right),
$$

which turns into the equation

$$
\lambda e^{-D(\lambda)} \Psi(\boldsymbol{t}, \mu)=-\left(\partial_{1}-\partial_{1} \log \Psi(\boldsymbol{t}, \lambda)\right) \Psi(\boldsymbol{t}, \mu)
$$

for the wave function

$$
\Psi(\boldsymbol{t}, z)=\frac{\tau\left(\boldsymbol{t}-\left[z^{-1}\right]\right)}{\tau(\boldsymbol{t})} e^{\xi(\boldsymbol{t}, z)}
$$

(66) may be thought of as a generating functional form of auxiliary linear equations for $\Psi(\boldsymbol{t}, z)$. Let us introduce the fundamental Schur functions $h_{j}(\boldsymbol{t}), j=0,1,2, \ldots$, by the generating function

$$
e^{\xi(\boldsymbol{t}, z)}=\sum_{j=0}^{\infty} h_{j}(\boldsymbol{t}) z^{j} .
$$

(66) can be thereby decomposed to the linear equations

$$
h_{j}\left(-\tilde{\partial}_{\boldsymbol{t}}\right) \Psi(\boldsymbol{t}, \mu)=f_{j}(\boldsymbol{t}) \Psi(\boldsymbol{t}, \mu) \quad(j=2,3, \ldots),
$$

where $\tilde{\partial}_{\boldsymbol{t}}$ denotes the vector

$$
\tilde{\partial}_{\boldsymbol{t}}=\left(\partial_{1}, \frac{1}{2} \partial_{2}, \cdots, \frac{1}{n} \partial_{n}, \ldots\right)
$$


of derivative operators, and $f_{j}(\boldsymbol{t})$ 's are the coefficients of Laurent expansion of $\partial_{1} \log \Psi(\boldsymbol{t}, z)$ at $z=\infty$, i.e.,

$$
\partial_{1} \log \Psi(\boldsymbol{t}, z)=z+\partial_{1}\left(e^{-D(z)}-1\right) \log \tau(\boldsymbol{t})=z+\sum_{j=1}^{\infty} f_{j+1}(\boldsymbol{t}) z^{-j} .
$$

It is well known that these Laurent coefficients give conserved densities of the KP hierarchy.

We can convert (66) to linear equations of the familiar evolutionary form

$$
\partial_{n} \Psi(\boldsymbol{t}, z)=B_{n} \Psi(\boldsymbol{t}, z) \quad(n=1,2, \ldots),
$$

where $B_{n}$ is a differential operator of the form

$$
B_{n}=\partial_{1}^{n}+b_{n 2} \partial_{1}^{n-2}+\cdots+b_{n n} .
$$

This is achieved by the following method [2, Appendix C]. Let us rewrite (66) as

$$
\left(1-e^{-D(\lambda)}\right) \Psi(\boldsymbol{t}, \mu)=X(\boldsymbol{t}, \lambda) \Psi(\boldsymbol{t}, \mu),
$$

where $X(\lambda)$ denotes the differential operator

$$
X(\boldsymbol{t}, \lambda)=\lambda^{-1} \partial_{1}+1-\lambda^{-1} \partial_{1} \log \Psi(\boldsymbol{t}, \lambda)
$$

that depends on $\lambda$. Let $X_{k}(\boldsymbol{t}, \lambda), k=2,3, \ldots$, be differential operators defined by the recurrence relation

$X_{k+1}(\boldsymbol{t}, \lambda)=X_{k}(\boldsymbol{t}, \lambda)-X_{k}\left(\boldsymbol{t}-\left[\lambda^{-1}\right], \lambda\right)\left(1-X_{k}(\boldsymbol{t}, \lambda)\right), \quad X_{1}(\boldsymbol{t}, \lambda)=X(\boldsymbol{t}, \lambda)$.

$X_{k}(\boldsymbol{t}, \lambda)$ is a differential operator of the form

$$
X_{k}(\boldsymbol{t}, \lambda)=\lambda^{-k} \partial_{1}^{k}+\cdots,
$$

and turns out to satisfy the equation

$$
\left(1-e^{-D(\lambda)}\right)^{k} \Psi(\boldsymbol{t}, \mu)=X_{k}(\boldsymbol{t}, \lambda) \Psi(\boldsymbol{t}, \mu) .
$$

Moreover, by construction,

$$
X_{k}(\lambda)=O\left(\lambda^{-k}\right) .
$$

Consequently, the action of

$$
D(\lambda)=-\log \left(1-\left(1-e^{-D(\lambda)}\right)\right)=\sum_{k=1}^{\infty} \frac{1}{k}\left(1-e^{-D(\lambda)}\right)^{k}
$$

on $\Psi(\boldsymbol{t}, \mu)$ is well defined, and we have the linear equation

$$
D(\lambda) \Psi(\boldsymbol{t}, \mu)=\sum_{k=1}^{\infty} \frac{1}{k} X_{k}(\boldsymbol{t}, \lambda) \Psi(\boldsymbol{t}, \mu),
$$

which, upon expanded in powers of $\lambda$, gives the usual auxiliary linear equations (69) as expected. 


\subsection{Generating functional form of auxiliary linear equa- tion for multi-component case}

We now turn to the the multi-component case. Let us recall the following form of the differential Fay identities first derived from the bilinear equations (54):

$$
\begin{aligned}
& \frac{\tau\left(\boldsymbol{s}, \boldsymbol{t}+\left[\lambda^{-1}\right]_{0}+\left[\mu^{-1}\right]_{0}\right) \tau(\boldsymbol{s}, \boldsymbol{t})}{\tau\left(\boldsymbol{s}, \boldsymbol{t}+\left[\lambda^{-1}\right]_{0}\right) \tau\left(\boldsymbol{s}, \boldsymbol{t}+\left[\mu^{-1}\right]_{0}\right)} \\
& =1+\frac{1}{\lambda-\mu} \partial_{01} \log \frac{\tau\left(\boldsymbol{s}, \boldsymbol{t}+\left[\mu^{-1}\right]_{0}\right)}{\tau\left(\boldsymbol{s}, \boldsymbol{t}+\left[\lambda^{-1}\right]_{0}\right)}, \\
& \lambda \frac{\tau\left(\boldsymbol{s}-\boldsymbol{e}_{0}+\boldsymbol{e}_{\alpha}, \boldsymbol{t}+\left[\lambda^{-1}\right]_{0}+\left[\mu^{-1}\right]_{\alpha}\right) \tau(\boldsymbol{s}, \boldsymbol{t})}{\tau\left(\boldsymbol{s}, \boldsymbol{t}+\left[\lambda^{-1}\right]_{0}\right) \tau\left(\boldsymbol{s}-\boldsymbol{e}_{0}+\boldsymbol{e}_{\alpha}, \boldsymbol{t}+\left[\mu^{-1}\right]_{\alpha}\right)} \\
& =\lambda+\partial_{01} \log \frac{\tau\left(\boldsymbol{s}-\boldsymbol{e}_{0}+\boldsymbol{e}_{\alpha}, \boldsymbol{t}+\left[\mu^{-1}\right]_{\alpha}\right)}{\tau\left(\boldsymbol{s}, \boldsymbol{t}+\left[\lambda^{-1}\right]_{0}\right)}, \\
& \frac{\tau\left(\boldsymbol{s}-2 \boldsymbol{e}_{0}+2 \boldsymbol{e}_{\alpha}, \boldsymbol{t}+\left[\lambda^{-1}\right]_{\alpha}+\left[\mu^{-1}\right]_{\alpha}\right) \tau(\boldsymbol{s}, \boldsymbol{t})}{\tau\left(\boldsymbol{s}-\boldsymbol{e}_{0}+\boldsymbol{e}_{\alpha}, \boldsymbol{t}+\left[\lambda^{-1}\right]_{\alpha}\right) \tau\left(\boldsymbol{s}-\boldsymbol{e}_{0}+\boldsymbol{e}_{\alpha}, \boldsymbol{t}+\left[\mu^{-1}\right]_{\alpha}\right)} \\
& =\frac{\lambda \mu}{\lambda-\mu} \partial_{01} \log \frac{\tau\left(\boldsymbol{s}-\boldsymbol{e}_{0}+\boldsymbol{e}_{\alpha}, \boldsymbol{t}+\left[\mu^{-1}\right]_{\alpha}\right)}{\tau\left(\boldsymbol{s}-\boldsymbol{e}_{0}+\boldsymbol{e}_{\alpha}, \boldsymbol{e}+\left[\lambda^{-1}\right]_{\alpha}\right)}, \\
& \epsilon_{\alpha \beta} \frac{\tau\left(\boldsymbol{s}-2 \boldsymbol{e}_{0}+\boldsymbol{e}_{\alpha}+\boldsymbol{e}_{\beta}, \boldsymbol{t}+\left[\lambda^{-1}\right]_{\alpha}+\left[\mu^{-1}\right]_{\beta}\right) \tau(\boldsymbol{e}, \boldsymbol{t})}{\tau\left(\boldsymbol{s}-\boldsymbol{e}_{0}+\boldsymbol{e}_{\alpha}, \boldsymbol{t}+\left[\lambda^{-1}\right]_{\alpha}\right) \tau\left(\boldsymbol{s}-\boldsymbol{e}_{0}+\boldsymbol{e}_{\beta}, \boldsymbol{t}+\left[\mu^{-1}\right]_{\beta}\right)} \\
& =\partial_{01} \log \frac{\tau\left(\boldsymbol{s}-\boldsymbol{e}_{0}+\boldsymbol{e}_{\beta}, \boldsymbol{t}+\left[\mu^{-1}\right]_{\beta}\right)}{\tau\left(\boldsymbol{s}-\boldsymbol{e}_{0}+\boldsymbol{e}_{\alpha}, \boldsymbol{t}+\left[\lambda^{-1}\right]_{\alpha}\right)} .
\end{aligned}
$$

We now convert these equations to auxiliary linear equations for the scalarvalued wave functions

$$
\begin{aligned}
& \Psi_{0}(\boldsymbol{s}, \boldsymbol{t}, z)=\frac{\tau\left(\boldsymbol{s}, \boldsymbol{t}-\left[z^{-1}\right]_{0}\right)}{\tau(\boldsymbol{s}, \boldsymbol{t})} z^{s_{0}} e^{\xi\left(\boldsymbol{t}_{0}, z\right)} \\
& \Psi_{\beta}(\boldsymbol{s}, \boldsymbol{t}, z)=\tilde{\epsilon}_{\beta}(\boldsymbol{s}) \frac{\tau\left(\boldsymbol{s}+\boldsymbol{e}_{0}-\boldsymbol{e}_{\beta}, \boldsymbol{t}-\left[z^{-1}\right]_{\beta}\right)}{\tau(\boldsymbol{s}, \boldsymbol{t})} z^{s_{\beta}} e^{\xi\left(\boldsymbol{t}_{\beta}, z\right)} .
\end{aligned}
$$

To simplify notations, these wave functions are referred to as $\Psi_{0}(z)$ and $\Psi_{\beta}(z)$. We have the following analogue of (66) for these wave functions.

Theorem 3. (70)-(73) can be converted to the linear equations

$$
\begin{aligned}
\lambda e^{-D(\lambda)} \Psi(\mu) & =-\left(\partial_{01}-\partial_{01} \log \Psi_{0}(\lambda)\right) \Psi(\mu), \\
e^{-\partial_{\alpha 0}-D_{\alpha}(\lambda)} \Psi(\mu) & =\left(\partial_{01}-\partial_{01} \log \Psi_{\alpha}(\lambda)\right) \Psi(\mu) \quad(\alpha=1, \ldots, N)
\end{aligned}
$$

for $\Psi(\mu)=\Psi_{0}(\mu), \Psi_{1}(\mu), \ldots, \Psi_{N}(\mu)$. 
Proof. Let us first consider (70). This equation has the same structure as the differential Fay identity of the KP hierarchy. Therefore we can repeat the foregoing calculations for the KP hierarchy to derive the equation

$$
\lambda e^{-D_{0}(\lambda)} \Psi_{0}(\mu)=-\left(\partial_{01}-\partial_{01} \Psi_{0}(\lambda)\right) \Psi_{0}(\mu),
$$

which is nothing but (75) for $\Psi(\mu)=\Psi_{0}(\mu)$.

As regards (71), we shift the variables as

$$
\boldsymbol{s} \rightarrow \boldsymbol{s}+\boldsymbol{e}_{0}-\boldsymbol{e}_{\alpha}, \quad \boldsymbol{t} \rightarrow \boldsymbol{t}-\left[\lambda^{-1}\right]-\left[\mu^{-1}\right]
$$

and consider the equation

$$
\begin{aligned}
& \lambda \frac{\tau\left(\boldsymbol{s}+\boldsymbol{e}_{0}-\boldsymbol{e}_{\alpha}, \boldsymbol{t}-\left[\lambda^{-1}\right]_{0}-\left[\mu^{-1}\right]_{\alpha}\right) \tau(\boldsymbol{s}, \boldsymbol{t})}{\tau\left(\boldsymbol{s}, \boldsymbol{t}-\left[\lambda^{-1}\right]_{0}\right) \tau\left(\boldsymbol{s}+\boldsymbol{e}_{0}-\boldsymbol{e}_{\alpha}, \boldsymbol{t}-\left[\mu^{-1}\right]_{\alpha}\right)} \\
& =\lambda+\partial_{01} \log \frac{\tau\left(\boldsymbol{s}, \boldsymbol{t}-\left[\lambda^{-1}\right]_{0}\right)}{\tau\left(\boldsymbol{s}+\boldsymbol{e}_{0}-\boldsymbol{e}_{\alpha}, \boldsymbol{t}-\left[\mu^{-1}\right]_{\alpha}\right)}
\end{aligned}
$$

thus obtained. We can rewrite this equation in two different ways as

$$
\begin{aligned}
& \lambda e^{-\partial_{\alpha 0}-D_{\alpha}(\mu)} \frac{\tau\left(\boldsymbol{s}, \boldsymbol{t}-\left[\lambda^{-1}\right]_{0}\right)}{\tau(\boldsymbol{s}, \boldsymbol{t})} \\
& =\frac{\tau\left(\boldsymbol{s}, \boldsymbol{t}-\left[\lambda^{-1}\right]_{\alpha}\right)}{\tau(\boldsymbol{s}, \boldsymbol{t})}\left(\lambda+\partial_{01} \log \frac{\tau\left(\boldsymbol{s}+\boldsymbol{e}_{0}-\boldsymbol{e}_{\alpha}, \boldsymbol{t}-\left[\lambda^{-1}\right]_{0}\right)}{\tau\left(\boldsymbol{s}, \boldsymbol{t}-\left[\mu^{-1}\right]_{\alpha}\right)}\right)
\end{aligned}
$$

and

$$
\begin{aligned}
& \lambda e^{-D_{0}(\lambda)} \frac{\tau\left(\boldsymbol{s}+\boldsymbol{e}_{0}-\boldsymbol{e}_{\alpha}, \boldsymbol{t}-\left[\mu^{-1}\right]_{\alpha}\right)}{\tau(\boldsymbol{s}, \boldsymbol{t})} \\
& =\frac{\tau\left(\boldsymbol{s}+\boldsymbol{e}_{0}-\boldsymbol{e}_{\alpha}, \boldsymbol{t}-\left[\mu^{-1}\right]_{\alpha}\right)}{\tau(\boldsymbol{s}, \boldsymbol{t})}\left(\lambda+\partial_{01} \log \frac{\tau\left(\boldsymbol{s}+\boldsymbol{e}_{0}-\boldsymbol{e}_{\alpha}, \boldsymbol{t}-\left[\lambda^{-1}\right]_{0}\right)}{\tau\left(\boldsymbol{s}, \boldsymbol{t}-\left[\mu^{-1}\right]_{\alpha}\right)}\right) .
\end{aligned}
$$

Upon interchanging $\lambda$ and $\mu$, the first equation reduces to

$$
e^{-\partial_{\alpha 0}-D_{\alpha}(\lambda)} \Psi_{0}(\mu)=\left(\partial_{01}-\partial_{01} \log \Psi_{\alpha}(\lambda)\right) \Psi_{0}(\mu),
$$

which coincides with (76) for the case of $\Psi(\mu)=\Psi_{0}(\mu)$. On the other hand, the second equation becomes

$$
\lambda e^{-D_{0}(\lambda)} \Psi_{\alpha}(\mu)=-\left(\partial_{01}-\partial_{01} \log \Psi_{0}(\lambda)\right) \Psi_{\alpha}(\mu),
$$

yielding (75) for the case of $\Psi(\mu)=\Psi_{\alpha}(\mu)$.

We now turn to (72). By shifting the variables as

$$
\boldsymbol{s} \rightarrow \boldsymbol{s}+2 \boldsymbol{e}_{0}-2 \boldsymbol{e}_{\alpha}, \quad \boldsymbol{t} \rightarrow \boldsymbol{t}-\left[\lambda^{-1}\right]_{\alpha}-\left[\mu^{-1}\right]_{\alpha},
$$


we obtain the equation

$$
\begin{aligned}
& \frac{\tau\left(\boldsymbol{s}+2 \boldsymbol{e}_{0}-2 \boldsymbol{e}_{\alpha}, \boldsymbol{t}-\left[\lambda^{-1}\right]_{\alpha}-\left[\mu^{-1}\right]_{\alpha}\right) \tau(\boldsymbol{s}, \boldsymbol{t})}{\tau\left(\boldsymbol{s}+\boldsymbol{e}_{0}-\boldsymbol{e}_{\alpha}, \boldsymbol{t}-\left[\lambda^{-1}\right]_{\alpha}\right) \tau\left(\boldsymbol{s}+\boldsymbol{e}_{0}-\boldsymbol{e}_{\alpha}, \boldsymbol{t}-\left[\mu^{-1}\right]_{\alpha}\right)} \\
& =\frac{\lambda \mu}{\lambda-\mu} \partial_{01} \log \frac{\tau\left(\boldsymbol{s}+\boldsymbol{e}_{0}-\boldsymbol{e}_{\alpha}, \boldsymbol{t}-\left[\lambda^{-1}\right]_{\alpha}\right)}{\tau\left(\boldsymbol{s}+\boldsymbol{e}_{0}-\boldsymbol{e}_{\alpha}, \boldsymbol{t}-\left[\mu^{-1}\right]_{\alpha}\right)},
\end{aligned}
$$

equivalently,

$$
\begin{aligned}
& e^{-\partial_{\alpha 0}-D_{\alpha}(\lambda)} \frac{\tau\left(\boldsymbol{s}+\boldsymbol{e}_{0}-\boldsymbol{e}_{\alpha}, \boldsymbol{t}-\left[\mu^{-1}\right]_{\alpha}\right)}{\tau(\boldsymbol{s}, \boldsymbol{t})} \\
& =\frac{\tau\left(\boldsymbol{s}+\boldsymbol{e}_{0}-\boldsymbol{e}_{\alpha}, \boldsymbol{t}-\left[\mu^{-1}\right]_{\alpha}\right)}{\tau(\boldsymbol{s}, \boldsymbol{t})} \frac{\lambda \mu}{\lambda-\mu} \partial_{01} \log \frac{\tau\left(\boldsymbol{s}+\boldsymbol{e}_{0}-\boldsymbol{e}_{\alpha}, \boldsymbol{t}-\left[\lambda^{-1}\right]_{\alpha}\right)}{\tau\left(\boldsymbol{s}+\boldsymbol{e}_{0}-\boldsymbol{e}_{\alpha}, \boldsymbol{t}-\left[\mu^{-1}\right]_{\alpha}\right)} .
\end{aligned}
$$

We now use the difference relation

$$
\tilde{\epsilon}_{\alpha}\left(s+e_{0}-e_{\alpha}\right)=-\tilde{\epsilon}_{\alpha}(s)
$$

to convert the last equation to the linear equation

$$
e^{-\partial_{\alpha 0}-D_{\alpha}(\lambda)} \Psi_{\alpha}(\mu)=\left(\partial_{01}-\partial_{01} \log \Psi_{\alpha}(\lambda)\right) \Psi_{\alpha}(\mu)
$$

for $\Psi_{\alpha}(\mu)$. This is exactly (76) for the case of $\Psi(\mu)=\Psi_{\alpha}(\mu)$.

Lastly, we consider (73). Upon shifting variables as

$$
\boldsymbol{s} \rightarrow \boldsymbol{s}+2 \boldsymbol{e}_{0}-\boldsymbol{e}_{\alpha}-\boldsymbol{e}_{\beta}, \quad \boldsymbol{t} \rightarrow \boldsymbol{t}-\left[\lambda^{-1}\right]_{\alpha}-\left[\mu^{-1}\right]_{\beta},
$$

we have the equation

$$
\begin{aligned}
& \epsilon_{\alpha \beta} \frac{\tau\left(\boldsymbol{s}+2 \boldsymbol{e}_{0}-\boldsymbol{e}_{\alpha}-\boldsymbol{e}_{\beta}, \boldsymbol{t}-\left[\lambda^{-1}\right]_{\alpha}-\left[\mu^{-1}\right]_{\beta}\right) \tau(\boldsymbol{s}, \boldsymbol{t})}{\tau\left(\boldsymbol{s}+\boldsymbol{e}_{0}-\boldsymbol{e}_{\alpha}, \boldsymbol{t}-\left[\lambda^{-1}\right]_{\alpha}\right) \tau\left(\boldsymbol{s}+\boldsymbol{e}_{0}-\boldsymbol{e}_{\beta}, \boldsymbol{t}-\left[\mu^{-1}\right]_{\beta}\right)} \\
& =\partial_{01} \log \frac{\tau\left(\boldsymbol{s}+\boldsymbol{e}_{0}-\boldsymbol{e}_{\alpha}, \boldsymbol{t}-\left[\lambda^{-1}\right]_{\alpha}\right)}{\tau\left(\boldsymbol{s}+\boldsymbol{e}_{0}-\boldsymbol{e}_{\beta}, \boldsymbol{t}-\left[\mu^{-1}\right]_{\beta}\right)}
\end{aligned}
$$

equivalently,

$$
\begin{aligned}
& \epsilon_{\alpha \beta} e^{-\partial_{\alpha 0}-D_{\alpha}(\lambda)} \frac{\tau\left(\boldsymbol{s}+\boldsymbol{e}_{0}-\boldsymbol{e}_{\beta}, \boldsymbol{t}-\left[\mu^{-1}\right]_{\beta}\right)}{\tau(\boldsymbol{s}, \boldsymbol{t})} \\
& =\frac{\tau\left(\boldsymbol{s}+\boldsymbol{e}_{0}-\boldsymbol{e}_{\beta}, \boldsymbol{t}-\left[\mu^{-1}\right]_{\beta}\right)}{\tau(\boldsymbol{s}, \boldsymbol{t})} \partial_{01} \log \frac{\tau\left(\boldsymbol{s}+\boldsymbol{e}_{0}-\boldsymbol{e}_{\alpha}, \boldsymbol{t}-\left[\lambda^{-1}\right]_{\alpha}\right)}{\tau\left(\boldsymbol{s}+\boldsymbol{e}_{0}-\boldsymbol{e}_{\beta}, \boldsymbol{t}-\left[\mu^{-1}\right]_{\beta}\right)} .
\end{aligned}
$$

Noting the difference relation

$$
\tilde{\epsilon}_{\beta}\left(\boldsymbol{s}+\boldsymbol{e}_{0}-\boldsymbol{e}_{\alpha}\right)=-\epsilon_{\alpha \beta} \tilde{\epsilon}_{\beta}(\boldsymbol{s}),
$$

we can convert the last equation to the linear equation

$$
e^{-\partial_{\alpha 0}-D_{\alpha}(\lambda)} \Psi_{\beta}(\mu)=\left(\partial_{01}-\partial_{01} \log \Psi_{\alpha}(\lambda)\right) \Psi_{\beta}(\mu)
$$

for $\Psi_{\beta}(\mu)$. This gives (76) for $\Psi(\mu)=\Psi_{\beta}(\mu), \beta \neq \alpha$. Thus all equations of (75) and (76) have been derived from the multi-component differential Fay identities. 


\subsection{Evolutionary form of auxiliary linear equations}

We can convert the generating functional auxiliary linear equations (75)(76) to an "evolutionary" form. As it turns out below, those auxiliary linear equations turn out to be a mixture of those of one-component KP hierarchies and two-dimensional Toda field equations. The KP hierarchies live in each sector of the $N+1$ sets of time variables $\boldsymbol{t}_{0}, \boldsymbol{t}_{1}, \ldots, \boldsymbol{t}_{N}$. The Toda field equations (and, actually, the Toda hierarchies) connect the $\boldsymbol{t}_{0}$-sector with the other $N$ sectors pairwise. We can thus eventually recover all building blocks of the scalar Lax formalism of the multi-component KP hierarchy [26].

\subsubsection{KP hierarchy in $t_{0}$-sector}

Let us note that the first set (75) of these equations have the same structure as their counterpart (66) in the KP hierarchy. Therefore they can be converted to linear equations of the form

$$
\partial_{0 n} \Psi(z)=B_{0 n}\left(\partial_{01}\right) \Psi(z)
$$

for $\Psi(z)=\Psi_{0}(z), \Psi_{1}(z), \ldots, \Psi_{N}(z) . B_{0 n}\left(\partial_{01}\right)$ is a differential operator of the form

$$
B_{0 n}\left(\partial_{01}\right)=\partial_{01}^{n}+O\left(\partial_{01}^{n-2}\right) .
$$

Among the $N+1$ wave functions, $\Psi_{0}(z)$ plays the role of the wave function in the usual KP hierarchy. If we define the dressing operator

$$
W_{0}\left(\partial_{01}\right)=1+\sum_{j=1}^{\infty} w_{0 j} \partial_{01}^{-j}
$$

such that

$$
\Psi_{0}(z)=W_{0}\left(\partial_{01}\right) z^{s_{0}} e^{\xi\left(\boldsymbol{t}_{0}, z\right)}=\left(1+\sum_{j=1}^{\infty} w_{0 j} z^{-j}\right) z^{s_{0}} e^{\xi\left(\boldsymbol{t}_{0}, z\right)},
$$

the auxiliary linear equations for $\Psi_{0}(z)$ can be converted to the so called Sato equations

$$
\frac{\partial W_{0}\left(\partial_{01}\right)}{\partial t_{0 n}}=B_{0 n}\left(\partial_{01}\right) W_{0}\left(\partial_{01}\right)-W_{0}\left(\partial_{01}\right) \partial_{01}^{n}
$$

As usual, this implies that $B_{0 n}\left(\partial_{01}\right)$ is given by the KP-like formula

$$
B_{0 n}\left(\partial_{01}\right)=\left(L_{0}\left(\partial_{01}\right)^{n}\right)_{\geq 0},
$$


where $(\quad)_{\geq 0}$ denotes the projection to nonnegative powers of $\partial_{01}$, and $L_{0}\left(\partial_{01}\right)$ is the pseudo-differential Lax operator

$$
L_{0}\left(\partial_{01}\right)=W_{0}\left(\partial_{01}\right) \cdot \partial_{01} \cdot W_{0}\left(\partial_{01}\right)^{-1} \text {. }
$$

\subsubsection{Toda field equation in $\left(t_{0}, t_{\alpha}\right)$-sector}

We now turn to (76). As we show below, the auxiliary linear equations of Toda fields emerge in the lowest and next-to-lowest orders of expansion in powers of $\lambda$.

The Toda fields $\phi_{\beta}=\phi_{\beta}(\boldsymbol{s}, \boldsymbol{t})$ are defined in an exponentiated form as

$$
e^{\phi_{\beta}}=\tilde{\epsilon}_{\beta}(\boldsymbol{s}) \frac{\tau\left(\boldsymbol{s}+\boldsymbol{e}_{0}-\boldsymbol{e}_{\beta}, \boldsymbol{t}\right)}{\tau(\boldsymbol{s}, \boldsymbol{t})},
$$

which is actually the leading coefficients of the amplitude part of $\Psi_{\beta}(z)$, i.e.,

$$
\Psi_{\beta}(z)=\left(e^{\phi_{\beta}}+\sum_{j=1}^{\infty} w_{\beta j} z^{-j}\right) z^{s_{\beta}} e^{\xi\left(\boldsymbol{t}_{\beta}, z\right)} .
$$

Let us examine the lowest orders of expansion of (176) in powers of $\lambda$. The $\lambda^{0}$-terms yield a linear equation of the form

$$
e^{-\partial_{\alpha 0}} \Psi_{\beta}(z)=\left(\partial_{01}-\partial_{01} \phi_{\alpha}\right) \Psi_{\beta}(z) .
$$

( $\mu$ has been replaced by z.) Introducing a new field $q_{\alpha}$ as

$$
q_{\alpha}=\partial_{01} \phi_{\alpha}
$$

we can rewrite this equation as

$$
\partial_{01} \Psi_{\beta}(z)=\left(e^{-\partial_{\alpha 0}}+q_{\alpha}\right) \Psi_{\beta}(z)
$$

Let us note that the associated Hamilton-Jacobi equation

$$
\partial_{01} S_{\beta}(z)=e^{-\partial_{\alpha 0} S_{\beta}(z)}+q_{\alpha}
$$

in quasi-classical approximation is nothing but (22). The $\lambda^{1}$-terms of (76) give the somewhat ugly equation

$$
-e^{-\partial_{\alpha 0}} \partial_{\alpha 1} \Psi_{\beta}(z)=\partial_{01} \partial_{\alpha 1} \log \tau\left(\boldsymbol{s}+\boldsymbol{e}_{0}-\boldsymbol{e}_{\alpha}, \boldsymbol{t}\right) \cdot \Psi_{\beta}(z) .
$$

We can rewrite it as

$$
\partial_{\alpha 1} \Psi_{\beta}(z)=r_{\alpha} e^{\partial_{\alpha 0}} \Psi_{\beta}(z),
$$


where

$$
r_{\alpha}=-\partial_{01} \partial_{\alpha 1} \log \tau(\boldsymbol{s}, \boldsymbol{t}) .
$$

The associated Hamilton-Jacobi equation reads

$$
\partial_{\alpha 1} S_{\beta}(z)=r_{\alpha} e^{\partial_{\alpha 0} S_{\beta}(z)} .
$$

Using the foregoing Hamilton-Jacobi equation for the $t_{01}$ flow, we can rewrite it as

$$
\partial_{\alpha 1} S_{\beta}(z)=\frac{r_{\alpha}}{\partial_{01} S_{\beta}(z)-q_{\alpha}} .
$$

This coincides with (21) for $n=1$.

As one can thus see from quasi-classical approximation, the auxiliary fields $q_{\alpha}$ and $r_{\alpha}$ correspond to those of the universal Whitham hierarchy defined by (19). Moreover, substituting

$$
\Psi_{\alpha}(z)=\left(e^{\phi_{\alpha}}+O\left(z^{-1}\right)\right) z^{s_{\alpha}} e^{\xi\left(\boldsymbol{t}_{\alpha}, z\right)}
$$

in (83) for the case of $\beta=\alpha$ and extracting the coefficient of $z^{s_{\alpha}} e^{\xi\left(\boldsymbol{t}_{\alpha}, z\right)}$, we obtain another expression

$$
r_{\alpha}=\exp \left(\phi_{\alpha}(\boldsymbol{s}, \boldsymbol{t})-\phi_{\alpha}\left(\boldsymbol{s}-\boldsymbol{e}_{0}+\boldsymbol{e}_{\alpha}, \boldsymbol{t}\right)\right)
$$

of $r_{\alpha}$. This expression corresponds to (24).

(82) and (83) may be thought of as auxiliary linear equations of the two-dimensional Toda fields with continuous variables $t_{01}, t_{\alpha 1}$ and discrete variable $s_{\alpha}$. The zero-curvature equation

$$
\left[\partial_{01}-e^{-\partial_{\alpha 0}}-q_{\alpha}, \partial_{\alpha 1}-r_{\alpha} e^{\partial_{\alpha 0}}\right]=0
$$

comprises the two equations

$$
\begin{array}{r}
\partial_{01} r_{\alpha}(\boldsymbol{s}, \boldsymbol{t})-\left(q_{\alpha}(\boldsymbol{s}, \boldsymbol{t})-q_{\alpha}\left(\boldsymbol{s}-\boldsymbol{e}_{0}+\boldsymbol{e}_{\alpha}, \boldsymbol{t}\right)\right) r_{\alpha}(\boldsymbol{s}, \boldsymbol{t})=0, \\
\partial_{\alpha 1} q_{\alpha}(\boldsymbol{s}, \boldsymbol{t})+r_{\alpha}\left(\boldsymbol{s}+\boldsymbol{e}_{0}-\boldsymbol{e}_{\alpha}, \boldsymbol{t}\right)-r_{\alpha}(\boldsymbol{s}, \boldsymbol{t})=0 .
\end{array}
$$

The first equation of (86) is automatically satisfied under the definition of $q_{\alpha}$ and $r_{\alpha}$ by (81) and (85); the second equation reduces to the Toda field equation

$$
\begin{aligned}
\partial_{01} \partial_{\alpha 1} \phi_{\alpha}(\boldsymbol{s}, \boldsymbol{t}) & +\exp \left(\phi_{\alpha}\left(\boldsymbol{s}+\boldsymbol{e}_{0}-\boldsymbol{e}_{\alpha}, \boldsymbol{t}\right)-\phi_{\alpha}(\boldsymbol{s}, \boldsymbol{t})\right) \\
& -\exp \left(\phi_{\alpha}(\boldsymbol{s}, \boldsymbol{t})-\phi_{\alpha}\left(\boldsymbol{s}-\boldsymbol{e}_{0}+\boldsymbol{s}_{\alpha}, \boldsymbol{t}\right)\right)=0 .
\end{aligned}
$$

This explains why a multi-dimensional dispersionless Toda field equation (or the Boyer-Finley equation) shows up in the universal Whitham hierarchy 32 . 


\subsubsection{Auxiliary linear equation of Schrödinger type in $\left(t_{0}, t_{\alpha}\right)$-sector}

The $\left(\boldsymbol{t}_{0}, \boldsymbol{t}_{\alpha}\right)$-sector is also accompanied by an auxiliary linear equation of the form

$$
\left(\left(\partial_{01}-q_{\alpha}\right) \partial_{\alpha 1}-r_{\alpha}\right) \Psi(z)=0
$$

satisfied by $\Psi(z)=\Psi_{0}(z), \Psi_{1}(z), \ldots, \Psi_{N}(z)$. These are analogues of twodimensional "integrable Schrödinger equations" [34, 35].

(88) can be derived from the Toda-like auxiliary linear equations (82) and (83) as follows. We first apply the operator $\partial_{01}-q_{\alpha}$ to both hand sides of (83). This yields an equation of the form

$$
\begin{aligned}
\left(\partial_{01}-q_{\alpha}\right) \Psi_{\beta}(z) & =\left(\partial_{01}-q_{\alpha}\right)\left(r_{\alpha} e^{\partial_{\alpha 0}} \Psi_{\beta}(z)\right) \\
& =\left(\partial_{01} r_{\alpha}\right) e^{\partial_{\alpha 0}} \Psi_{\beta}(z)+r_{\alpha}\left(\partial_{01}-q_{\alpha}\right)\left(e^{\partial_{\alpha 0}} \Psi_{\beta}(z)\right) .
\end{aligned}
$$

By the first equation of (86), we can rewrite the first term in the last line as

$$
\left(\partial_{01} r_{\alpha}\right) e^{\partial_{\alpha 0}} \Psi_{\beta}(z)=\left(q_{\alpha}(\boldsymbol{s}, \boldsymbol{t})-q_{\alpha}\left(\boldsymbol{s}-\boldsymbol{e}_{0}+\boldsymbol{e}_{\alpha}, \boldsymbol{t}\right)\right) r_{\alpha} e^{\partial_{\alpha 0}} \Psi_{\beta}(z) .
$$

As regards the second term, we use (82) to rewrite its main part as

$$
\begin{aligned}
\left(\partial_{01}-q_{\alpha}\right)\left(e^{\partial_{\alpha 0}} \Psi_{\beta}(z)\right) & =e^{\partial_{\alpha 0}} \partial_{01} \Psi_{\beta}(z)-q_{\alpha} e^{\partial_{\alpha 0}} \Psi_{\beta}(z) \\
& =e^{\partial_{\alpha 0}}\left(e^{-\partial_{\alpha 0}}+q_{\alpha}\right) \Psi_{\beta}-q_{\alpha} \Psi_{\beta}(z) \\
& =\Psi_{\beta}(z)+\left(q_{\alpha}\left(\boldsymbol{s}-\boldsymbol{e}_{0}+\boldsymbol{e}_{\alpha}, \boldsymbol{t}\right)-q_{\alpha}(\boldsymbol{s}, \boldsymbol{t})\right) e^{\partial_{\alpha 0}} \Psi_{\beta}(z) .
\end{aligned}
$$

Thus the terms proportional to $q_{\alpha}(\boldsymbol{s}, \boldsymbol{t})-q_{\alpha}\left(\boldsymbol{s}-\boldsymbol{e}_{0}+\boldsymbol{e}_{\alpha}, \boldsymbol{t}\right)$ cancel each other, and (88) follows.

\subsubsection{KP hierarchies in other sectors}

Let us now consider (76). Applying the shift operator $e^{\partial_{\alpha 0}}$ to both hand side yields

$$
e^{-D_{\alpha}(\lambda)} \Psi(\mu)=\left(\partial_{01}-e^{\partial_{\alpha 0}} \partial_{01} \log \Psi_{\alpha}(\lambda)\right) e^{\partial_{\alpha 0}} \Psi(\mu) .
$$

On the other hand, (83) implies that

$$
e^{\partial_{\alpha 0}} \Psi(\mu)=r_{\alpha}^{-1} \partial_{\alpha 1} \Psi(\mu) .
$$

Therefore we can eliminate the difference term $e^{\partial_{\alpha 0}} \Psi(\mu)$ and obtain the differential equation

$$
e^{-D_{\alpha}(\lambda)} \Psi(\mu)=\left(\partial_{01}-e^{\partial_{\alpha 0}} \partial_{01} \log \Psi_{\alpha}(\lambda)\right) r_{\alpha}^{-1} \partial_{\alpha 1} \Psi(\mu) .
$$


Letting as $\lambda \rightarrow \infty$, we have

$$
\Psi(\mu)=\left(\partial_{01}-e^{\partial_{\alpha 0}} q_{\alpha}\right) r_{\alpha}^{-1} \partial_{\alpha 1} \Psi(\mu) .
$$

Taking the difference of these two equations and doing some algebra, we eventually obtain an equation of the form

$$
\left(1-e^{-D_{\alpha}(\lambda)}\right) \Psi(\mu)=X_{\alpha}(\lambda) \Psi(\mu),
$$

where $X_{\alpha}(\lambda)$ is the first-order differential operator

$$
X_{\alpha}(\lambda)=\left(\partial_{01} \log \frac{\tau\left(\boldsymbol{s}-\boldsymbol{e}_{0}+\boldsymbol{e}_{\alpha}, \boldsymbol{t}-\left[\lambda^{-1}\right]\right)}{\tau\left(\boldsymbol{s}-\boldsymbol{e}_{0}+\boldsymbol{e}_{\alpha}, \boldsymbol{t}\right)}\right) r_{\alpha}^{-1} \partial_{\alpha 1} .
$$

We can now repeat the reasoning in the derivation of (69) from (66). The outcome is a system of "evolutionary" linear equations of the form

$$
\partial_{\alpha n} \Psi(z)=B_{\alpha n}\left(\partial_{\alpha 1}\right) \Psi(z)
$$

where $B_{\alpha n}\left(\partial_{\alpha 1}\right)$ is an $n$-th order differential operator without 0 -th order term, i.e.,

$$
B_{\alpha n}\left(\partial_{\alpha 1}\right)=b_{\alpha n 0} \partial_{\alpha 1}^{n}+\cdots+b_{\alpha n n-1} \partial_{\alpha 1}
$$

The absence of 0 -th order term is a consequence of the structure of $X_{\alpha}(z)$. (69) may be interpreted as auxiliary linear equations of the one-component KP hierarchy in the $\boldsymbol{t}_{\alpha}$-sector.

\section{Conclusion}

We have thus identified the universal Whitham hierarchy of genus zero with the dispersionless limit of the charged multi-component KP hierarchy. In the course of this rather lengthy consideration, we have seen a number of remarkable aspects of these systems in themselves.

A main conclusion of this consideration is that the dispersionless Hirota equations and the differential Fay identities can play the role of master equations that characterize the (usual and dispersionless) $\tau$-functions of these integrable systems. This interpretation is complementary to the conventional point of view based on the Hamilton-Jacobi equations and the auxiliary linear equations. We have an honest impression that the approach from the Hirota equations is technically simpler and conceptually more essential. This approach deserves to be pursued further. 
Alongside these fundamental aspects, we expect some applications of the results of this paper. For instance, the recent paper [13] of Bonora et al. conjectures the relevance of a multi-component version of the disperesionless Toda hierarchy in light-cone string field theory. This conjecture might be explained in our framework. Another intriguing issue is to generalize the notion of associativity equations [9] to the multi-component setting. In this respect, we should mention the recent work of Konopelchenko and Magri [36], in which a novel approach to the universal Whitham hierarchy is discussed along with some other issues on dispersionless integrable systems.

\section{Acknowedgements}

We would like to thank A. Zabrodin for allowing us to contribute this paper. K.T. is grateful to B. Konopelchenko, L. Martínez Alonso, M. Mañas and A. Sorin for useful discussions during the SISSA conference "RiemannHilbert Problems, Integrability and Asymptotics" in September, 2005. This research was partially supported by Grant-in-Aid for Scientific Research No. 16340040, No. 18340061 and No. 18540210 from the Japan Society for the Promotion of Science.

\section{References}

[1] M. Jimbo and T. Miwa, Solitons and infinite dimensional Lie algebras, Publ. RIMS, Kyoto Univ., 19 (1983), 943-1001.

[2] K. Takasaki and T. Takebe, Integrable hierarchies and dispersionless limit, Rev. Math. Phys. 7 (1995), 743-808.

[3] M. Adler and P. van Moerbeke, A matrix integral solution to twodimensional $W_{p}$-gravity, Comm. Math. Phys. 147 (1992), 25-56.

[4] R. Carroll and Y. Kodama, Solutions of the dispersionless Hirota equations, J. Phys. A28 (1995), 6373-6378.

[5] P.B. Wiegmann and A. Zabrodin, Conformal maps and integrable hierarchies, Comm. Math. Phys. 213 (2000), 523-538.

[6] M. Mineev-Weinstein, P.B. Wiegmann and A. Zabrodin, Integrable structure of interface dynamics, Phys. Rev. Lett. 84 (2000), 5106-5109.

[7] I.K. Kostov, I. Krichever, M. Mineev-Weinstein, P.B. Wiegmann and A. Zabrodin, $\tau$-function for analytic curve, in "Random matrices and 
their applications," edited by P. Bleher and A. Its, MSRI Publ. vol. 40 (Cambridge University Press, Cambridge, 2001), pp. 285-299.

[8] A. Zabrodin, Dispersionless limit of Hirota equations in some problems of complex analysis, Teor. Mat. Fiz. 129 (2001), 239-257.

[9] A. Boyarsky, A. Marshakov, O. Ruchayskiy, P. Wiegmann and A. Zabrodin, Associativity equations in dispersionless integrable hierarchies, Phys. Lett. B 515 (2001), 483-492.

[10] A. Boyarsky and O. Ruchayskiy, Integrability in SFT and new representation of KP tau-function, JHEP 0303 (2003), 027.

[11] A. Boyarsky, B. Kulik and O. Ruchayskiy, String field theory vertices, integrability and boundary states, JHEP 0311 (2003), 045.

[12] L. Bonora and A.S. Sorin, Integrable structures in string field theory, Phys. Lett. B553 (2003), 317-324.

[13] L. Bonora, R.J. Scherer Santos, A.S. Sorin and D.D. Tolla, Light-cone Superstring Field Theory, pp-wave background and integrability properties, Class. Quant. Grav. 23 (2006), 799-816.

[14] L. Takhtajan, Free bosons and tau-functions for compact Riemann surfaces and closed smooth Jordan curves I, Lett. Math. Phys. 56 (2001), $181-228$.

[15] L.-P. Teo, Analytic functions and integrable hierarchies - characterization of tau functions, Lett. Math. Phys. 64 (2003), 75-92.

[16] L.-P. Teo, Fay-like identities of the Toda lattice hierarchy and its dispersionless limit, Rev. Math. Phys. 18 (2006), 1055-1074.

[17] L.V. Bogdanov and B.G. Konopelchenko, On dispersionless BKP hierarchy and its reductions, J. Nonlin. Math. Phys. 12 (2005), Supplement $1,64-75$.

[18] K. Takasaki, Dispersionless Hirota equations of two-component BKP hierarchy, SIGMA 2 (2006), Paper 057.

[19] Y.T. Chen and M.-H. Tu, On kernel formulas and dispersionless Hirota equations, J. Math. Phys. 47 (2006), 102702.

[20] T. Takebe, L.-P. Teo and A. Zabrodin, Löwner equations and dispersionless hierarchies, J. Phys. A: Math. Gen. 39 (2006), 11479-11501. 
[21] M. Sato, Soliton equations as dynamical systems on an infinite dimensional Grassmannian manifold, Surikaiseki Kenkyusho Kokyuroku vol. 439 (RIMS, Kyoto University, 1981), pp. 30-46.

[22] M. Sato and Y. Sato, Soliton equations as dynamical systems on an infinite dimensional Grassmannian manifold, in "Nonlinear PDE in Applied Science," edited by H. Fujita, P.D. Lax and G. Strang, Lect. Notes. Num. Anal. vol. 5 (Kinokuniya, Tokyo, 1983), pp. 259-271.

[23] E. Date, M. Jimbo, M. Kashiwara and T. Miwa, Transformation groups for soliton equations III, J. Phys. Soc. Japan 50 (1981), no. 11, 38063812 .

[24] L. Dickey, Soliton equations and Hamiltonian systems (World Scientific, Singapore, 1991).

[25] V. Kac and J. van de Leur, The $n$-component KP hierarchy and representation theory, in "Important developments in soliton theory", edited by A.S Fokas and V.E. Zakharov (Springer-Verlag, Berlin and Heidelberg, 1993).

[26] K. Takasaki, Dispersionless integrable hierarchies revisited, talk at the SISSA workshop "Riemann-Hilbert problems, integrability and asymptotics" (September, 2005). A pdf file of slides is available via the Internet (http://www.math.h.kyoto-u.ac.jp/ ${ }^{\sim}$ takasaki/res/sissa05slide.pdf). Unfortunately, the scalar Lax formalism therein contained a small error, namely, the sign factor $\tilde{\epsilon}_{\alpha}(\boldsymbol{s})$ was overlooked in the expression of wave functions.

[27] K. Ueno and K. Takasaki, Toda lattice hierarchy, Advanced Studies in Pure Math. vol. 4 (Kinokuniya, Tokyo, 1984), pp. 1-94

[28] I.M. Krichever, The $\tau$-function of the universal Whitham hierarchy, matrix models and topological field theories, Comm. Pure. Appl. Math. 47 (1994), 437-475.

[29] I.Krichever, M.Mineev-Weinstein, P.Wiegmann and A. Zabrodin, Laplacian Growth and Whitham Equations of Soliton Theory, Physica D198 (2004), 1-28.

[30] I.Krichever, A.Marshakov and A.Zabrodin, Integrable Structure of the Dirichlet Boundary Problem in Multiply-Connected Domains, Comm. Math. Phys. 259 (2005), 1-44. 
[31] F. Guil, M. Mañas and L. Mart'nnez Alonso, On the Whitham hierarchies: reductions and hodograph solutions, J. Phys. A36 (2003), 40474062 .

[32] M. Mañas, E. Medina and L. Martínez Alonso, On the Whitham hierarchy: dressing scheme, string equations and additional symmetries, J. Phys. A39 (2006), 2349-2382.

[33] L. Martínez Alonso, E. Medina and M. Mañas, String equations in Whitham hierarchies: $\tau$-functions and Virasoro constraints, e-print nlin.SI/0510001.

[34] S. Novikov and A. Veselov, Finite-gap two-dimensional periodic Schrödinger operators, Soviet Math. Dokl. 30 (1984), no. 3, 588-591, 705-708.

[35] I. Krichever, A characterization of Prym varieties, Int. Math. Res. Not. 2006 (2006), article ID 81476.

[36] B.G. Konopelchienko and F. Magri, Coisotropic deformations of associative algebras and dispersioness integrable hierarchies, Comm. Math. Phys. 274 (2007), 627-658. 